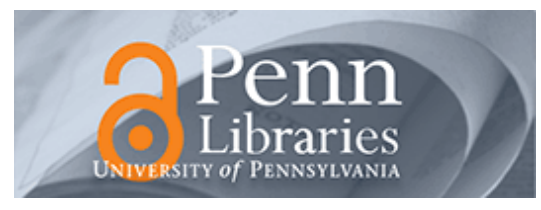

University of Pennsylvania

ScholarlyCommons

Finance Papers

Wharton Faculty Research

$3-2014$

\title{
Optimal Design of Social Comparison Effects: Setting Reference Groups and Reference Points
}

Guillaume Roels

Xuanming Su

University of Pennsylvania

Follow this and additional works at: https://repository.upenn.edu/fnce_papers

Part of the Finance and Financial Management Commons

\section{Recommended Citation}

Roels, G., \& Su, X. (2014). Optimal Design of Social Comparison Effects: Setting Reference Groups and Reference Points. Management Science, 60 (3), 606-627. http://dx.doi.org/10.1287/mnsc.2013.1760

This paper is posted at ScholarlyCommons. https://repository.upenn.edu/fnce_papers/120

For more information, please contact repository@pobox.upenn.edu. 


\title{
Optimal Design of Social Comparison Effects: Setting Reference Groups and Reference Points
}

\begin{abstract}
In this paper, we study how social planners should exploit social comparisons to pursue their objectives. We consider two modes of social comparison, referred to as behind-averse and ahead-seeking behaviors, depending on whether individuals experience a utility loss from underperforming or a utility gain from overperforming relative to their peers. Modeling social comparison as a game between players, we find that ahead-seeking behavior leads to output polarization, whereas behind-averse behavior leads to output clustering. A social planner can mitigate these effects in two ways: (i) by providing the full reference distribution of outputs instead of an aggregate reference point based on the average output and (ii) by assigning players into uniform rather than diverse reference groups. Social planners may thus need to tailor the reference structure to the predominant mode of social comparison and their objective. A performance-focused social planner may set the reference structure so as to maximize the output of either the top or the bottom player depending on whether she puts greater marginal weight to larger or smaller outputs. When the social planner also cares about utility, she faces a dilemma because performance optimization may not be aligned with utility maximization. Inevitably, the social planner will have to confront equity issues because better performance may not reflect greater effort or greater ability.
\end{abstract}

\section{Keywords}

social comparisons, reference points, behavioral operations, noncooperative game theory

\section{Disciplines}

Business | Finance and Financial Management 


\title{
Optimal Design of Social Comparison Effects: Setting Reference Groups and Reference Points
}

\author{
Guillaume Roels \\ UCLA Anderson School of Management, 110 Westwood Plaza, Los Angeles, CA 90095, USA, groels@anderson.ucla.edu \\ Xuanming $\mathrm{Su}$ \\ The Wharton School, University of Pennsylvania, 3730 Walnut Street, Philadelphia, PA 19104, USA, \\ xuanming@wharton.upenn.edu
}

\begin{abstract}
In this paper, we study how social planners should exploit social comparisons to pursue their objectives. We consider two modes of social comparison, referred to as behind-averse and ahead-seeking behaviors, depending on whether individuals experience a utility loss from under-performing or a utility gain from overperforming relative to their peers. Modeling social comparison as a game between players, we find that aheadseeking behavior leads to output polarization whereas behind-averse behavior leads to output clustering. A social planner can mitigate these effects in two ways, (i) by providing the full reference distribution of outputs instead of an aggregate reference point based on the average output, and (ii) by assigning players into uniform rather than diverse reference groups. Social planners may thus need to tailor the reference structure to the predominant mode of social comparison and their objective. A performance-focused social planner may set the reference structure so as to maximize the output of either the top or the bottom player depending on whether she puts greater marginal weight to larger or smaller outputs. When the social planner also cares about utility, she faces a dilemma because performance-optimization may not be aligned with utility-maximization. Inevitably, the social planner will have to confront equity issues because better performance may not reflect greater effort or greater ability.
\end{abstract}

Key words: social comparisons, reference points, behavioral operations, non-cooperative game theory History:

\section{Introduction}

In many social contexts, people exhibit a natural tendency to compare themselves against their peers. Furthermore, the amount of effort individuals put into an activity may be driven by analogous investments made by their peers. For example, disclosing relative pay information has a significant impact on workers' economic output (Blanes i Vidal and Nossol 2011, Netessine and Yakubovich 2012), providing relative performance feedback influences the academic performance of high school students (Azmat and Iriberri 2008), and reporting households' relative energy usage has a significant effect on energy consumption rates (Schultz et al. 2007). As a consequence, social comparisons can have a profound impact on outcomes. 
Figure 1 Social comparisons to induce energy conservancy.

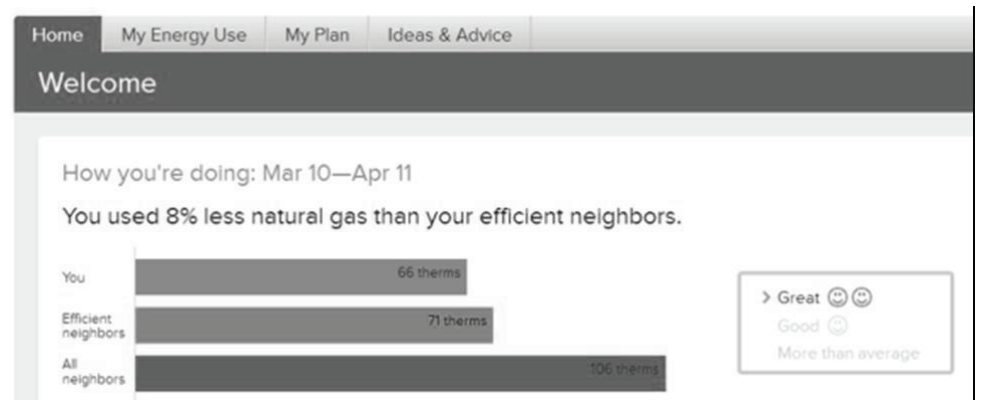

Note. http://blog.ucsusa.org/smiley-faces-vs-vampires-knowledge-about-power-is-power/. Retrieved on January 10, 2013.

When making comparisons to others, people may focus on those who perform better (e.g., Collins 1996) or those who perform worse (e.g., Wills 1981). In this paper, we distinguish between two contrasting modes of social comparison. On one hand, people may enjoy utility from overperforming relative to others, and on the other, people may face disutility from under-performing relative to others. By our terminology, people may be ahead-seeking or behind-averse. Depending on the contextual, cultural, or institutional environment, each mode of social comparison may dominate. For example, in some educational systems, students strive to be at the top of their class (Stevenson et al., 1990). A student who achieved a perfect score on a test may derive pleasure out of doing better than other students who scored less; the same perfect score yields less utility if the entire class has the same achievement. On the contrary, social comparisons induced to reduce alcohol consumption on college campuses (Lewis and Neighbors 2008) appeal to students' behindaverse behavior since it is unlikely that any student would enjoy utility gains purely from drinking less alcohol than reported statistics. In our analysis below, we demonstrate that ahead-seeking behavior and behind-averse behavior influence outcomes in significantly different ways.

Central to any social comparison process is the reference structure, which consists of a particular reference group and specific pieces of information about that group. For example, Figure 1 depicts an energy bill that benchmarks a customer's energy consumption to its neighbors. Comparisons could be made across all houses of the neighborhood, or only across houses of similar size. We say that the reference group is diverse in the former alternative and uniform in the latter alternative. Diverse reference groups may exert pressure on larger households to conserve as much energy as smaller households, while uniform reference groups may maintain competitive pressure by placing everyone on equal footing. Furthermore, notice from Figure 1 that the customer has two reference points: energy consumption of all its neighbors and of its most efficient neighbors. In general, the information provided may range from a brief summary statistic such as the group average to detailed information about every individual in the reference group. We refer to the former as an 
aggregate reference point and the latter as the full reference distribution. A single aggregate statistic channels each customer's attention onto whether he is more or less efficient relative to the statistic, whereas the full reference distribution allows for more nuanced comparisons with others who are more efficient as well as others who are less efficient. Therefore, the diversity of reference groups (i.e., diverse or uniform) and the granularity of reference points (i.e., aggregate or full) are two key features that shape the impact of social comparison effects. Our goal in this paper is to understand how to design the reference structure, defined as a combination of the two features above.

Careful design of the reference structure allows the social planner to influence individual choices. People are free to choose, but in the spirit of libertarian paternalism, the social planner plays the role of a "choice architect" and may seek to align individual choices with social objectives (Thaler and Sunstein 2008). ${ }^{1}$ In general, we adopt a broad perspective on the notion of a social planner, which includes service providers, employers, and policy makers. The social planner may have different goals. First, a common objective is to optimize the overall performance of the group. For instance, a utility company may want households to lower energy consumption, or a firm may seek high worker productivity. Alternatively, a social planner may wish to "develop stars." This certainly applies to Ph.D. programs that seek student placement records at top academic institutions. In contrast, for early-stage education (e.g., levels K-12), governments may opt for the "no child left behind" ideology and focus on the other end of the distribution; similar sentiments may prevail in health care services. Aside from performance considerations, a social planner, such as a service provider (e.g., a yoga teacher), may also care about individual utilities, especially in settings where customer retention is a direct function of their satisfaction. Finally, fairness concerns may also matter. For instance, outcomes should be commensurate with ability and effort. Ideally, the social planner would like to maintain equity and maximize everyone's utility while optimizing performance.

To explore the ideas above, we develop a static game-theoretic model with complete information in which each player chooses an output level (e.g., test score). Higher outputs are valuable but costly to each individual player. Instead of individual decision problems, we have to consider a noncooperative game between the players because with social comparisons, players' utilities depend on others' outputs. We undertake a systematic analysis by considering all four combinations of reference structures, i.e., with an aggregate reference point (based on the group's average output) or the full reference distribution, and with diverse or uniform reference groups.

${ }^{1}$ What people think is best for themselves may diverge from what is objectively best. Examples of time inconsistency and self-control problems abound in health maintenance (DellaVigna and Malmendier 2006) and retirement planning (Thaler and Benartzi 2004). 
Our results offer guidelines for social planners depending on their objectives and on whether social comparisons manifest through ahead-seeking or behind-averse behavior. Specifically, our analysis yields three main results. First, we find that social comparison creates incentives for players to cluster their outputs together in behind-averse environments and to choose polar extremes (i.e., either very high or very low outputs) in ahead-seeking environments. We refer to these effects as clustering and polarization of outputs.

Second, we show that a social planner can mitigate these social comparison effects by providing the full reference distribution and/or using more uniform reference groups. The polarization effect characterizing ahead-seeking environments stimulates over-achievers to perform better but leads to a worse performance from the under-achievers. In contrast, the clustering effect characterizing behind-averse environments encourages under-performers to work harder at the expense of the highperformers shirking in complacency. Depending on whether the social planner seeks to "develop stars" or to "leave no one behind," it may be more desirable to foster or mitigate those polarization and clustering effects. In particular, we find that a social planner who gives greater marginal weight to larger (smaller) outputs may focus her attention on the top- (bottom-)performing player. Therefore, the social planner needs to tailor the reference structure to her objective and to the predominant mode of social comparison.

Third, our results caution that it is never possible to attain perfection on the dimension of "customer satisfaction." In ahead-seeking environments, measures adopted to stimulate player output also increase player utility, so there may be complaints against "favoritism" by the players who derive the least utility and also perform the worst. In behind-averse environments, increases in equilibrium outputs are accompanied by lower utilities, so players who perform well may not even be happy in the social planner's "boot camps." In addition, outcomes may be perceived as unfair and unjust: some players may perform better than others who work harder or others with higher intrinsic "abilities." Therefore, there are many scenarios in which some players will be dissatisfied, and the social planner should be aware of such possibilities.

The rest of this paper is organized as follows. Section 2 provides a literature review. Section 3 describes our model and $\S 4$ derives the equilibrium outcomes. Sections 5 and 6 discuss outputoriented and utility-oriented strategies in manipulating reference points. Section 7 considers the impact of noisy observations of output. Finally, $\S 8$ concludes. All proofs appear in Appendix A and in an electronic companion.

\section{Literature Review}

Social comparisons have been studied in many domains, such as social psychology, behavioral economics, and operations management. We next review these streams of literature. 
In a seminal paper, Festinger (1954) proposes that people engage in social comparisons to fulfill their desire for self-evaluation: in the absence of objective standards, people compare themselves with others to evaluate their own opinions and abilities. Although Festinger's initial theory postulates that people tend to compare themselves with similar others, there is a large body of work on upward comparisons with others who are better off (e.g., Collins 1996) and downward comparisons with others who are worse off (e.g., Wills 1981). Both upward and downward comparisons may generate positive or negative emotions (Buunk et al. 1990). People may focus on similarities or differences (Brewer and Weber 1994) and may try to identify with targets of upward comparison but contrast against targets of downward comparisons (Lockwood et al. 2002). Social comparisons are in fact important drivers of one's happiness (Baucells and Sarin 2012). In this paper, we attempt to capture the above aspects of social comparison processes. By considering ahead-seeking and behind-averse preferences, we incorporate both upward and downward social comparisons and accommodate both positive and negative effects on utility. A comprehensive introduction to the literature on social comparisons can be found in Wood (1989) and Suls and Wheeler (2000).

In behavioral economics, there is a large body of work on social preferences. People exhibit social preferences when they care not only about material self-interest, but also about the economic outcomes of others. One prominent area of research on social preferences is in fairness and inequity aversion. People view disparities as unfair and seek to minimize them. This can be modeled using utility functions that penalize agents when their outcomes deviate from others' (e.g., Fehr and Schmidt 1999, Charness and Rabin 2002) or when their shares of the total pie deviate from the equal allocation benchmark (e.g., Bolton and Ockenfels 2000). Our model corresponds to the above in behind-averse environments, where agents who are behind aim to minimize disparities from others who are better; however, in ahead-seeking environments, agents who are ahead aim to increase differences by being further ahead. Another broad type of social preferences arises when people's well-being depend directly on others' payoffs. This dependence may be positive for altruists or negative for spiteful or envious individuals (see Andreoni 1990, Levine 1998). Consistent with spiteful behavior but contrary to altruistic behavior, our utility model implies that one is never better off when others' outcomes are improved; however, our results carry distinct interpretations from the behavioral underpinnings of altruism and spite. Nevertheless, we adopt a reference-based approach (Kahneman and Tversky 1979), similar to many models above, in which everyone else in the group serves as a separate and endogenous reference point.

Social comparisons lead individuals to benchmark their performance to their peers. Kandel and Lazear (1992) develop a general model of peer pressure and show that peer effects improve performance in partnerships, consistent with Festinger's idea that "a person who runs more slowly than others with whom he compares himself ... may spend considerable time practising running. 
In a similar situation where the ability in question is intelligence, the person may study harder" (Festinger 1954, p. 126). There is a stream of literature that provides empirical evidence for peer effects on labor productivity. In a laboratory experiment, Falk and Ichino (2006) show that peer effects increase productivity even when subjects were paid independently of their work output. Mas and Moretti (2009) use high-frequency scanner data to show that the productivity of supermarket cashiers increases when highly productive personnel is introduced into the same shift. Blanes i Vidal and Nossol (2011) report an increase in productivity after workers who were paid piece rates receive information about their own rank in the distribution of pay (and thus productivity). In a field experiment with a Dutch retail chain, Delfgaauw et al. (2012) find that merely providing rank information about relative sales performance at the store level led to an approximately $5 \%$ increase in sales growth; interestingly, adding monetary incentives did not generate any further improvement. Consistent with the above findings, we consider a model in which social comparisons lead to increased performance.

However, there is some evidence that social comparisons may also lead to decreased performance. For example, Bandiera et al. (2012) find that the productivity of teams of fruit pickers declines when team rankings are revealed. The negative impact arises because the introduction of rank incentives induced individuals to team up with partners of similar abilities, thereby skewing the distribution of team performance. Similarly, Barankay (2012) reports that rank feedback is associated with lower performance among furniture salespeople. Here, the negative effect is primarily due to agents' lack of knowledge about how much effort is required to achieve a certain rank, leading to demoralization in case one's performance is lower than expected. The effects above are arguably the most prominent in ordinal comparisons since differences in ranks are not representative of the magnitude of the difference in outputs. However, in this paper, we focus on cardinal rather than ordinal comparisons.

Incentive mechanisms based on relative performance evaluation have been widely studied. In labor economics, Lazear and Rosen (1981) show that rank-order tournaments are a useful alternative to piece-rate contracts when output cannot be directly or efficiently measured. Rosen (1986) extend these ideas to multiple rounds of elimination tournaments and show that in order to maintain performance incentives for survivors at every round, prizes for top ranks need to be very large, akin to CEO salaries. In operations management, Siemsen et al. (2007) show that relative performance evaluations are desirable when the outcomes of different tasks assigned to different agents are linked. In behavioral economics, Blanes i Vidal and Nossol (2011) study rank feedback in the absence of explicit financial incentives, noting that this is akin to tournaments without prizes where workers compete for relative position and the resulting productivity gain is achieved at no cost to the firm. In a similar vein, we do not consider financial incentives in this paper. 
There is abundant evidence on the impact of social comparisons in a wide range of application areas, including academic performance (Blanton et al. 1999, Azmat and Iriberri 2010), energy conservancy (Schultz et al. 2007, Ayres et al. 2012, Delmas and Lessem 2012), curbside recycling (Schultz 1998), and binge drinking (Lewis and Neighbors 2008); see Thaler and Sunstein (2008) for other applications. By and large, the studies above are concerned about the aggregate effect of social comparisons. In contrast, by identifying the clustering and polarization effects of social comparisons, we focus on the dispersion (or variance) of outcomes across individuals. Furthermore, the reference structure considered in existing papers is often study-specific and fixed at the outset. For instance, in energy conservancy programs, Schultz et al. (2007) report only the aggregate energy usage and do not appear to control for house size, whereas Ayres et al. (2012) provide electricity use statistics of "efficient neighbors" and "comparable neighbors." Taking a different approach, we consider a variety of reference structures and study their effect on output dispersion.

Notably, our research question differs by considering social comparisons as a mechanism that must be designed, or engineered. Social comparisons act in reference to some benchmark, and we posit that a social planner may have some flexibility regarding how to set that benchmark. The importance of framing reference points has been well documented both in laboratory experiments (Kahneman and Tversky 1979) and in natural experiments (Hossain and List 2012). Similarly, the mechanism design question in our paper can be viewed as setting up the appropriate reference points for social comparison. To our knowledge, this theme has been explored by two papers. First, in a laboratory setting, Kuhnen and Tymula (2012) find that organizations can improve employee productivity by providing feedback about relative rankings. Moreover, people with high ranks work harder over time but people at the bottom do not improve as much, suggesting that the latter should be assigned to new reference groups. The authors conjecture that there exists an optimal feedback policy but do not characterize it; we take on this task in this paper. Second, Carrell et al. (2013) conduct a field experiment with entering freshmen at the United States Air Force Academy. Using an assignment algorithm that was intended to maximize academic achievement, low-ability students were placed into squadrons with a high fraction of high-ability students. However, endogenous sorting of low and high ability students into separate social networks resulted in decreased performance relative to the control where students were randomly assigned. Although the outcome was negative, the results point to the importance of designing an optimal mechanism to reap the full benefits of peer effects. In this paper, we develop a theoretical framework to facilitate mechanism design for social comparisons.

In operations management, there is a handful of papers on the theme of social comparisons. Loch and $\mathrm{Wu}(2008)$ use laboratory experiments with human subjects to show that profit comparisons 
between a retailer and a manufacturer in a supply chain can lead them to deviate from profitmaximizing decisions, and surprisingly, Cui et al. (2007) show that when such comparisons are in effect, the simple wholesale price contract can coordinate the supply chain. Recently, Avc1 et al. (2012) studied the effect of social comparisons between two newsvendors and established results analogous to our clustering and polarization effects. So far, this literature has studied social comparisons between a pair of players, and our paper takes a step toward exploring social comparisons within a group of players. In group environments, there is more than one possible target of comparison, and we study how the social planner should actively influence the reference structure through an appropriate choice of reference groups and reference points.

In line with the recent interest in modeling consumer behavior (e.g., Shen and Su 2007; Netessine and Tang 2009), there are several papers that study how comparative effects between consumers can drive operational practices. Tereyagoglu and Veeraraghavan (2012) analyze production decisions when consumers engaging in conspicuous consumption value exclusivity (i.e., when others are excluded from consumption), while Veeraraghavan and Debo (2011) study the queueing implications of herding behavior (i.e., when others also join in consumption). Similar to the above, our paper joins the operations management literature and explores the impact of social comparisons.

Finally, a stream of research in operations management studies reference effects that arise when people look back at past outcomes. In particular, Popescu and Wu (2007) and Nasiry and Popescu (2011) study dynamic pricing strategies with reference effects, respectively when the reference price smoothly "acclimates" to price changes and when it is based on the "peak-end" history of prices. Aflaki and Popescu (2010) study how to manage long-term relationships when customers' satisfaction adapts to past service experiences, and Caro and Martinez-de-Albeniz (2012) consider pricing and product design decisions with satiation effects. In contrast to these studies, we focus on social contexts where reference points are endogenously determined by the actions of other players.

\section{3. $\quad$ Model}

Let us first consider an individual player $i$ who must choose a particular output $x_{i}$. Higher outputs are valuable but come at a cost. For example, students can learn the course material better by working harder, and athletes can attain a higher level of proficiency by training harder. We summarize all costs and benefits using a strictly concave (net) value function $V_{i}\left(x_{i}\right)$, so there is a unique output level that maximizes net value.

Next, let us consider a group of $n$ players. Players have a tendency to compare their outputs relative to others. ${ }^{2}$ Let $x_{0}$ be some reference output. We consider two modes of social comparisons.

\footnotetext{
${ }^{2}$ Alternatively, players may compare other performance metrics $\tilde{x}_{i}$ such as rewards or costs increasing in the output $x_{i}$. We can adapt our model to this case by assuming that players choose $\tilde{x}_{i}$ directly. The same analysis applies as long as players' net value is concave in $\tilde{x}_{i}$.
} 
First, each player $i$ may face disutility from achieving a lower output compared to $x_{0}$. In this case, we model the utility function of player $i$ following Fehr and Schmidt (1999) as

$$
U_{i}\left(x_{i}\right)=V_{i}\left(x_{i}\right)-\beta_{0}\left[x_{0}-x_{i}\right]^{+},
$$

in which $[x]^{+} \equiv \max (x, 0)$. For every unit of output that player $i$ is behind the reference point, the corresponding disutility is $\beta_{0} \geq 0$. In this case, we refer to the term $\beta_{0}\left[x_{0}-x_{i}\right]^{+}$as the behind-loss and we say that player $i$ is behind-averse. Alternatively, player $i$ may enjoy increased utility from achieving a higher output compared to $x_{0}$. The corresponding utility function of player $i$ is

$$
U_{i}\left(x_{i}\right)=V_{i}\left(x_{i}\right)+\alpha_{0}\left[x_{i}-x_{0}\right]^{+} .
$$

Similar to above, player $i$ enjoys utility of $\alpha_{0} \geq 0$ for every unit of output higher than the reference point. Here, we refer to the term $\alpha_{0}\left[x_{i}-x_{0}\right]^{+}$as the ahead-gain and we say that player $i$ is aheadseeking. Without loss of generality, we assume that each player is either purely ahead-seeking or purely behind-averse. ${ }^{3}$ In this model, utility gains or losses on top of the net value function $V_{i}\left(x_{i}\right)$ are brought about by social comparisons relative to a reference point $x_{0}$.

The social planner can actively influence the reference point $x_{0}$ for social comparisons. A natural candidate for $x_{0}$ is the average output of the group $\overline{\mathbf{x}} \equiv \sum_{j} x_{j} / n$. This is commonly observed in many applications (e.g., Figure 1). We model this case by using $x_{0}=\overline{\mathbf{x}}$ and setting $\alpha_{0}=n \alpha, \beta_{0}=n \beta$ to reflect the fact that this aggregate reference point is based on the choices of all $n$ players. In other words, the utility functions for ahead-seeking and behind-averse players are respectively

$$
U_{i}^{(\alpha a)}\left(x_{i} ; \mathbf{x}_{-i}\right)=V_{i}\left(x_{i}\right)+n \alpha \cdot\left[x_{i}-\overline{\mathbf{x}}\right]^{+} \text {and } U_{i}^{(\beta a)}\left(x_{i} ; \mathbf{x}_{-i}\right)=V_{i}\left(x_{i}\right)-n \beta \cdot\left[\overline{\mathbf{x}}-x_{i}\right]^{+},
$$

where we write $U_{i}\left(x_{i} ; \mathbf{x}_{-i}\right)$ to emphasize the dependence on player $i$ 's utility on others' outputs and its superscript refers to whether players are ahead-seeking (' $\alpha$ ') or behind-averse (' $\beta$ ') and to the fact that they receive an aggregate reference point (' $a$ ').

Alternatively, the social planner can stimulate social comparisons relative to a full reference distribution (' $f$ ') by making the individual outputs of each player readily available. We model this case by assuming that each individual output is a separate reference point and the associated parameters are $\alpha_{0}=\alpha, \beta_{0}=\beta$. In other words, the utility functions are, respectively for aheadseeking and behind-averse players,

$$
U_{i}^{(\alpha f)}\left(x_{i} ; \mathbf{x}_{-i}\right)=V_{i}\left(x_{i}\right)+\alpha \sum_{j}\left[x_{i}-x_{j}\right]^{+} \text {and } U_{i}^{(\beta f)}\left(x_{i} ; \mathbf{x}_{-i}\right)=V_{i}\left(x_{i}\right)-\beta \sum_{j}\left[x_{j}-x_{i}\right]^{+} .
$$

\footnotetext{
${ }^{3}$ Although ahead-seeking and behind-averse behaviors are distinct psychological phenomena, this framework can potentially model individuals as having both ahead-seeking $\left(\alpha_{0}>0\right)$ and behind-averse $\left(\beta_{0}>0\right)$ preferences. Any player with $\alpha_{0}>\beta_{0}$ can be modeled as purely ahead-seeking, and vice versa.
} 
Our analysis will study how the granularity of reference points interacts with social comparisons by contrasting these two extreme cases.

At a higher level, the social planner can influence the composition of the reference group. Should the reference group be diverse or uniform? To address this question, we consider two player types, $\theta=A, B .{ }^{4}$ All type- $\theta$ players have identical preferences and possess the value function $V_{\theta}(\cdot)$. Further, we assume that $V_{A}^{\prime}(x+k)=V_{B}^{\prime}(x)$. Given the same marginal value, a type- $A$ player is thus naturally predisposed to achieve $k$ more units of output than a type- $B$ player. For brevity, we drop the subscript and write $V_{B}(x) \equiv V(x)$. The utility of a type- $\theta$ player $i$ is denoted by $U_{\theta, i}\left(x_{i} ; \mathbf{x}_{-i}\right)$. Let $n_{\theta}$ denote the number of players of type $\theta$ in a reference group of size $n$, so $n_{A}+n_{B}=n$. We say that a group is uniform if all members are of the same type and diverse otherwise. In our equilibrium characterization, we consider diverse groups with arbitrary values of $n_{A}>0$ and $n_{B}>0$. However in $\S 5$, in order to study the impact of group diversity while keeping group size constant, ${ }^{5}$ we will contrast the following two setups: (i) two diverse reference groups, each consisting of $n_{A}=n / 2$ type- $A$ and $n_{B}=n / 2$ type- $B$ players, and (ii) two uniform reference groups, one with $n$ type- $A$ players and another with $n$ type- $B$ players. In either setup, the social planner serves two sections of $n$ players and there are $n$ players of each type. For instance, a professor could teach two sections of the same class to a hybrid population of master and undergraduate students, or teach one section to master students and one section to undergraduate students. Customers make social comparisons within their group but not across groups, so we analyze the player-game separately for each group.

Our analysis considers two different environments: in ahead-seeking environments, all players are ahead-seeking, while in behind-averse environments, all players are behind-averse. ${ }^{6}$ The parameter $\alpha \geq 0$ in an ahead-seeking environment or $\beta \geq 0$ in a behind-averse environment is common across every player and represents the strength of the social comparison effects in the particular context.

In summary, we consider eight different game configurations, depending on whether players are ahead-seeking or behind-averse, whether they have an aggregate reference point or a full reference distribution, and whether their reference group is uniform or diverse. For each configuration, we formulate a static game with complete information. ${ }^{7}$ In this game, all players $i=1, \ldots, n$ simultaneously choose an output $x_{i}$, following which all outputs are observed and players receive payoffs

${ }^{4}$ The denomination of types has been chosen in reference to the letter grades in academic contexts given the prevalence of social comparisons in education (Blanton et al. 1999, Azmat and Iriberri 2010).

${ }^{5}$ The effect of group size on social comparisons is in general indeterminate. For instance, Bond (2005) concludes from a meta-analysis that the effect of majority size on conformity can be either positive or negative.

${ }^{6}$ There may be situations with both ahead-seeking and behind-averse players in the same reference group. We characterize the equilibrium arising from such a situation in Appendix B.1 and show that the fundamental insights obtained with the extreme cases remain preserved.

${ }^{7}$ Our one-shot game is a simplification that ignores dynamic reference effects. For example, people may change their behavior in response to how they compared to peers in the past or to achieve more favorable relative standings in the future. In steady state, where everyone has full information (or rational expectations) about other's outputs, these dynamic considerations collapse into the static comparisons in our model. 
according to the utility functions $U_{i}\left(x_{i} ; \mathbf{x}_{-i}\right)$ specified above. We solve for the pure-strategy Nash equilibrium $\mathbf{x}^{*}$ such that $x_{i}^{*}=\arg \max _{x_{i}} U_{i}\left(x_{i} ; \mathbf{x}_{-i}^{*}\right)$. These equilibrium outputs reflect how players will behave in the presence of social comparison effects.

Without loss of generality, we restrict the strategy sets of each type- $\theta$ player to an interval $\left[L_{\theta}, R_{\theta}\right]$. To see this, consider an ahead-seeking (resp. behind-averse) type- $\theta$ player $i$ and suppose that there are $\tilde{n}_{i}$ other players with lower (resp. higher) outputs. Let us denote player $i$ 's best response by $C_{\theta}^{\alpha}\left(\tilde{n}_{i}\right)$ (resp. $C_{\theta}^{\beta}\left(\tilde{n}_{i}\right)$ ), which is increasing in $\tilde{n}_{i}$. For instance with the full reference distribution, an ahead-seeking player $i$ 's best response solves $V_{\theta}^{\prime}\left(x_{i}\right)+\tilde{n}_{i} \alpha=0$ (i.e., the first-order condition). Since there are $n$ players in the reference group, $\tilde{n}_{i}$ must be between 0 and $n-1$. Therefore, the best response of any ahead-seeking player must lie between $L_{\theta}^{\alpha} \equiv C_{\theta}^{\alpha}(0)$ and $R_{\theta}^{\alpha} \equiv$ $C_{\theta}^{\alpha}(n-1)$ and that of any behind-averse player must lie between $L_{\theta}^{\beta} \equiv C_{\theta}^{\beta}(0)$ and $R_{\theta}^{\beta} \equiv C_{\theta}^{\beta}(n-1)$. For brevity, we omit the superscripts and write $L_{\theta}, R_{\theta}$. We furthermore assume that the strategy sets of type- $A$ and type- $B$ players do not overlap, i.e., the difference between player types $(k)$ is so large that $R_{B}<L_{A} \cdot{ }^{8}$ Here, the interpretation is that players of different types exhibit large innate differences whereas players with small differences can be pooled into the same type.

Finally, we introduce some notation. We write player outputs in vector notation $\mathbf{x}=\left(\mathbf{x}_{B}, \mathbf{x}_{A}\right)$, in which $\mathbf{x}_{\theta}=\left(x_{\theta, 1}, \ldots, x_{\theta, n_{\theta}}\right)$ for $\theta=A, B$ and each $\mathbf{x}_{\theta}$ is sorted in ascending order. We use bars to denote averages, as in $\overline{\mathbf{x}}=\sum_{i=1}^{n} x_{i} / n$. In the event that all type- $\theta$ players choose the same output, we use $x_{\theta}$ as a shorthand for their output, i.e., we write $x_{\theta}=x$ instead of $\mathbf{x}_{\theta}=(x, \ldots, x)$. Finally, we use the standard notations $[x]^{+} \equiv \max (x, 0)$ and $\mathbb{1}_{[x>0]}=1$ if $x>0$ and zero otherwise.

\section{Equilibrium Characterization}

In this section, we characterize the pure-strategy Nash equilibrium outputs $\mathbf{x}^{*}$ in the game. To illustrate the following results, consider a cohort with $n_{A}=3$ type- $A$ players and $n_{B}=$ 4 type- $B$ players, with value function $V_{B}(x)=V_{A}(x+15)=100 x-x^{2}$. All players are either ahead-seeking or behind-averse with $\alpha=\beta=5$. Under these parameters, type- $B$ players choose their outputs between $L_{B}=50$ and $R_{B}=65$, and type- $A$ players choose them between $L_{A}=70$ and $R_{A}=85$. When players are ahead-seeking, the equilibrium outcome is $\mathbf{x}^{(\alpha a)}=$ $(50,50,50,50,85,85,85)$ with an aggregate reference point and $\mathbf{x}^{(\alpha f)}=(50,52.5,55,57.5,80,82.5,85)$ with the full reference distribution. When players are behind-averse, the equilibrium outcome is $\mathbf{x}^{(\beta a)}=(65,65,65,65,70,70,70)$ with an aggregate reference point and one possible equilibrium outcome is $\mathbf{x}^{(\beta f)}=(62.6,62.5,62.5,62.5,73.75,73.75,73.75)$ with the full reference distribution.

In this example, the equilibrium output is distributed on two points, except when players are ahead-seeking and receive the full reference distribution, in which case no pair of players chooses

\footnotetext{
${ }^{8}$ We relax this assumption in Appendix B.2. The basic analysis in the main text remains qualitatively unchanged.
} 
the same output. Moreover, the outcome tends to be more clustered when players are behind-averse and more polarized when they are ahead-seeking, and this clustering or polarization effect is more heavily pronounced when players have an aggregate reference point than when they have the full reference distribution. As we show next, these observations tend to hold true in general.

We characterize the equilibrium structure first for a behind-averse environment and then for an ahead-seeking environment. In each environment, we first consider the case where the social planner sets an aggregate reference point and then the case where the social planner provides the full reference distribution. Our results hold for general group compositions (with $n_{A}$ type- $A$ players and $n_{B}$ type- $B$ players) and can be applied to both diverse reference groups (i.e., $n_{A}, n_{B}>0$ ) and uniform reference groups (either $n_{A}=0$ or $n_{B}=0$ ).

Mathematically, the game is supermodular, i.e., outputs are strategic complements, when players are behind-averse and submodular, i.e., outputs are strategic substitutes, when they are aheadseeking. Intuitively, behind-averse players tend to seek "safety in numbers" because they can avoid falling behind by staying close to their peers' output level. In contrast, ahead-seeking players want to pull ahead of their peers to enjoy ahead-gains.

We first consider the case with behind-averse players. We present our results in two separate propositions, first with an aggregate reference point and then with the full reference distribution.

Proposition 1. Suppose players are behind-averse and have an aggregate reference point.

(i) If $n_{A} \geq 1$ and $n_{B} \geq 1$, there is a unique pure-strategy Nash equilibrium with $x_{A}^{*}=L_{A}, x_{B}^{*}=R_{B}$.

(ii) If $n_{\theta}=n$, then for any $z_{\theta} \in\left[C_{\theta}^{\beta}(0), C_{\theta}^{\beta}(n-1)\right], x_{\theta}^{*}=z_{\theta}$ is a Nash equilibrium.

When players are behind-averse and have an aggregate reference point, they thus choose their outputs at the inner boundaries of their action spaces when the group is diverse. Referring back to the introductory numerical example, $\mathbf{x}^{(\beta a)}=(65,65,65,65,70,70,70)$.

Proposition 2. Suppose players are behind-averse and have the full reference distribution. For any $z_{A} \in\left[C_{A}^{\beta}(0), C_{A}^{\beta}\left(n_{A}-1\right)\right]$ and $z_{B} \in\left[C_{B}^{\beta}\left(n_{A}\right), C_{B}^{\beta}(n-1)\right], x_{A}^{*}=z_{A}, x_{B}^{*}=z_{B}$ is a Nash equilibrium.

In Propositions 1(ii) and 2 above, there are multiple equilibria. For such cases, we shall use standard risk-dominance arguments to focus on one particular equilibrium. ${ }^{9}$ Specifically, the selected equilibrium in Proposition 1(ii) satisfies $V_{\theta}^{\prime}\left(x_{\theta}\right)+\beta(n-1) / 2=0$; and the selected equilibrium in Proposition 2 satisfies $V_{A}^{\prime}\left(x_{A}\right)+\beta\left(n_{A}-1\right) / 2=0$ and $V_{B}^{\prime}\left(x_{B}\right)+\beta n_{A}+$

\footnotetext{
${ }^{9}$ When the state space is restricted to be discrete, the proposed equilibrium turns out to be $\mathbf{1} / \mathbf{2}$-dominant (Kajii and Morris 1997) and, if $n_{A}=0$, the unique $u$-dominant equilibrium (Kojima 2006). These dominance concepts extend the risk-dominance concept to $n$-player games with $m>2$ actions and share some of its axiomatic foundations axiomatic (Harsanyi and Selten 1988) and stability properties (Kojima 2006). In $\S 7$, we also show that, when performance metrics are noisy, the equilibrium converges to this selected equilibrium as the noise tends to zero.
} 
$\beta\left(n_{B}-1\right) / 2=0$. In the introductory numerical example, that selected equilibrium is $\mathbf{x}^{(\beta f)}=$ $(62.6,62.5,62.5,62.5,73.75,73.75,73.75)$.

Now, we turn to ahead-seeking players. Although existence of a pure-strategy Nash equilibrium is in general not guaranteed in submodular games (Vives 1999), we establish its existence in our model. Similar to above, we consider first the case with an aggregate reference point and then the case with the full reference distribution.

The next proposition shows that, with an aggregate reference point, the equilibrium outputs of ahead-seeking players are polarized at the two extreme ends: $L_{\theta}$ and $R_{\theta}$. In the introductory numerical example, $\mathbf{x}^{(\alpha a)}=(50,50,50,50,85,85,85)$.

Proposition 3. Suppose players are ahead-seeking and have an aggregate reference point. There is a unique pure-strategy Nash equilibrium, in which $m_{\theta}$ type- $\theta$ players choose $L_{\theta}$ and $n_{\theta}-m_{\theta}$ of the remaining type- $\theta$ players choose $R_{\theta}$, for $\theta=A, B$. There exist constants $l \in\left[L_{B}, R_{B}\right]$ and $r \in\left[L_{A}, R_{B}\right]$ (so $L_{B}<l<R_{B}<L_{A}<r<R_{A}$ ) such that:

(i) If $\frac{n_{A} R_{A}+n_{B} L_{B}}{n} \in\left[L_{B}, l\right)$, then $m_{A}=0$ and $m_{B} \in\left[1, n_{B}-1\right]$.

(ii) If $\frac{n_{A} R_{A}+n_{B} L_{B}}{n} \in(l, r)$, then $m_{A}=0$ and $m_{B}=n_{B}$.

(iii) If $\frac{n_{A} R_{A}+n_{B} L_{B}}{n} \in\left(r, R_{A}\right]$, then $m_{A} \in\left[1, n_{A}-1\right]$ and $m_{B}=n_{B}$.

In particular when the reference group consists of an equal number of each type of players (i.e., when $n_{A}=n_{B}=n / 2$ ), Proposition 3(ii) shows that all type- $A$ players choose $R_{A}$ and all type- $B$ players choose $L_{B}$ in equilibrium. There is thus polarization of outputs at the two extremes. This phenomenon persists even when the reference group is uniform. For example, when $n_{B}=n$ and $n_{A}=0$, Proposition 3(i) shows that polarization occurs within the action set of the representative type because some players choose the lowest possible output $\left(L_{B}\right)$ while the remaining players choose the highest possible output $\left(R_{B}\right)$.

Next, we consider the case in which social comparisons are based on the full reference distribution. In that case, it turns out that no pair of players chooses the same output (see Lemma A-4 in Appendix A). In the introductory numerical example, $\mathbf{x}^{(\alpha f)}=(50,52.5,55,57.5,80,82.5,85)$. The intuition is as follows: Suppose that it is optimal for a player to choose output $z$ and be ahead of $\ell$ other players. Then, it is not optimal for any other player to choose the same output $z$ and also be ahead of $\ell$ players since choosing an infinitesimally smaller output would increase her utility by increasing her value (since $V^{\prime}(z)<0$ ) while remaining ahead of $\ell$ players.

Proposition 4. Suppose players are ahead-seeking and have the full reference distribution. There is a unique pure-strategy Nash equilibrium $\mathbf{x}^{*}$ satisfying $V_{A}^{\prime}\left(x_{A, i}^{*}\right)+\left(n_{B}+i-1\right) \alpha=0$ for $i=1, \ldots, n_{A}$, and $V_{B}^{\prime}\left(x_{B, i}^{*}\right)+(i-1) \alpha=0$ for $i=1, \ldots, n_{B}$. 
Figure 2 Equilibrium outputs in a behind-averse environment.

\section{Full reference distribution}
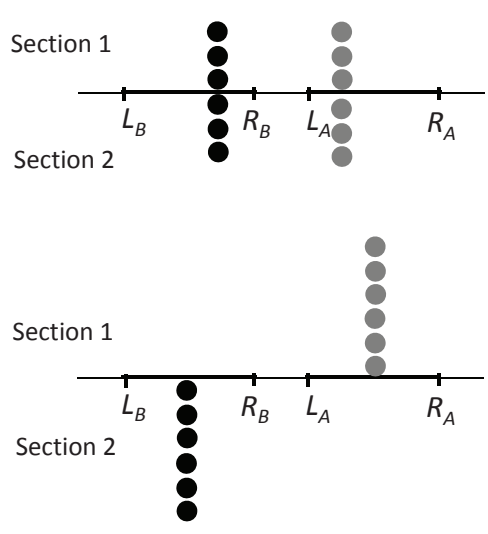

Ahead-seeking players thus tend to spread their outputs with the full reference distribution. Whereas type- $B$ players tend to choose outputs near the lower boundary of their strategy space $\left(L_{B}\right)$, type- $A$ players choose outputs near the upper boundary of their strategy space $\left(R_{A}\right)$. Within a given type, players tend to spread their outputs uniformly.

The results of Propositions 1-4 are illustrated in Figures 2 and 3, respectively for the cases of behind-averse and ahead-seeking players. We consider two sections of size $n$. With diverse reference groups, each section consists of an equal number of type- $A$ and type- $B$ players (i.e., $n_{A}=n_{B}=n / 2$ ). With uniform reference groups, section 1 consists of $n$ type- $A$ players and section 2 consists of $n$ type- $B$ players. Each plot shows a histogram of the players' outputs in equilibrium: Each circle represents a single player's output choice. Circles are stacked on top of one another when multiple players choose the same output. When players choose different outputs, circles are spread apart horizontally. Equilibrium choices are shown relative to the bounds $L_{\theta}$ and $R_{\theta}$.

Comparing Figures 2 and 3 reveals that outputs tend to be clustered together in behind-averse environments and polarized in ahead-seeking environments, consistent with the super- or submodular nature of the corresponding game. In Appendix B, we show that these equilibrium structural results remain valid when the reference group consists of both ahead-seeking and behind-averse types, and when the strategy sets of type- $A$ and type- $B$ players overlap. Specifically, we find that behind-averse players tend to cluster together; and if the strategy sets of type- $A$ and type- $B$ players overlap, all behind-averse players across all types choose the same output. We also find that ahead-seeking players always choose polarized outputs generally anchored at the upper boundary of the action set for type- $A$ players and the lower boundary of the action set for type- $B$ players, with or without dispersion depending on whether they have the full reference distribution or an 
Figure 3 Equilibrium outputs in an ahead-seeking environment.

\section{Full reference distribution}
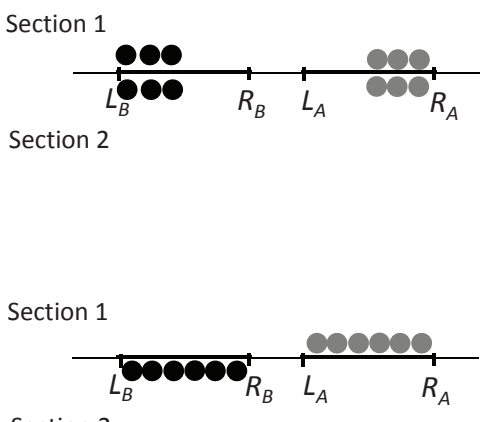

Section 2

aggregate reference point. Hence, the insights obtained with our base model carry over to more complex settings.

In addition, the figures reveal that the clustering effect when players are behind-averse and the polarization effect when they are ahead-seeking are the strongest when comparisons are based on an aggregate reference point obtained from a diverse reference group (i.e., top-left corner of each figure). This setting corresponds, in a way, to the simplest setting for the social planner. In comparison, the other settings require more effort from the social planner, such as classifying players according to their types to create uniform reference groups, or publishing the full vector of outputs to provide a full reference distribution. By taking these additional measures, the social planner can mitigate the clustering and polarization effects induced by social comparison. Henceforth, we use the term reference structure to refer to each of the four settings shown in Figures 2 and 3.

\section{Output-Focused Strategies}

Which reference structure should the social planner choose if she is concerned about players' outputs? The answer depends on her objective function. In this section, we assume that the social planner's objective is increasing and additively separable in output. Formally, we denote the social planner's objective as $\sum_{i=1}^{n} W\left(x_{i}\right)$, in which $W(x)$ is nondecreasing. We first consider the case where $W(x)$ is linear, i.e., the social planner maximizes the total output, assuming a quadratic value function $V(x) \cdot{ }^{10}$

\footnotetext{
${ }^{10}$ In Appendix B.3.2, a numerical study shows that the equilibrium structures described in Propositions 1-4 remain valid for a wide range of value functions $V(x)$. In the subsequent analysis, we sometimes focus, for the sake of tractability, on the representative case of a quadratic value function.
} 
Proposition 5 (Total Output). Suppose that the value function is quadratic. Then, the social planner, maximizing the total output $\left(\sum_{i=1}^{n_{A}} x_{A, i}+\sum_{i=1}^{n_{B}} x_{B, i}\right)$, should provide an aggregate reference point in ahead-seeking environments and provide the full reference distribution in behind-averse environments if and only if $n_{A} \geq n_{B}$; and, she is indifferent about the level of diversity of the reference group.

The above proposition shows that by manipulating the reference point granularity, the social planner can influence total output in groups. On the other hand, the composition of the reference group does not matter.

We can compare the equilibrium outputs of individual players using the results from Propositions 1-4. We find that in ahead-seeking environments, the outputs of type- $A$ (type- $B$ ) players are higher (lower) with an aggregate reference point than with the full reference distribution, and with diverse reference groups than with uniform reference groups; the above results are reversed in behindaverse environments. See Figures 2 and 3 for an illustration. Therefore, the reference structure has a different impact on the outputs of each player type, as summarized in Table 1. (Details are provided in Lemmas A-5 and A-6 in Appendix A.) Observe that the level of granularity of information that maximizes total output is the same as the one that maximizes the outputs of players who are in majority. For instance, in ahead-seeking environments, setting an aggregate reference point maximizes the total output when $n_{A} \geq n_{B}$ and it also maximizes the output of type- $A$ players. Hence, majority rules.

Table 1 Reference structures that maximize the output of type- $\theta$ players

\begin{tabular}{|c|c|c|}
\hline Objective & Ahead-seeking environment & Behind-averse environment \\
\hline \hline To increase $x_{A}:$ & diverse ref. group, aggregate ref. point & uniform ref. group, full ref. distribution \\
\hline To increase $x_{B}:$ & uniform ref. group, full ref. distribution & diverse ref. group, aggregate ref. point \\
\hline
\end{tabular}

To illustrate that result, consider a charity that wants to encourage donations. Consistent with the "warm glow effect" (Andreoni 1990), the predominant mode of social comparison is aheadseeking. Moreover, the charity faces more median-income (type- $B$ ) than high-income (type- $A$ ) potential donors, by the Pareto law of wealth distribution. According to Proposition 5, the charity should provide finely-grained information about the distribution of donations (while respecting donors' privacy) such as categorizing donations into tiers (McCardle et al. 2009).

Apart from equally considering all players' outputs, the social planner could pursue other objectives and give more weight to either the highest or the lowest outputs. In particular, the social planner may care more about the extremes, such as the upper or lower tails of the distribution of player outputs. We model such considerations using either a convex objective function $W(x)$, which 
emphasizes the importance of the right tail, or a concave objective function $W(x)$, which emphasizes the importance of the left tail. When $W(x)$ is convex, the social planner (e.g., a tennis academy) benefits more from developing a few "stars" (e.g., tennis champions) than from marginally raising the output level of a large pool of low-performing players. By contrast when $W(x)$ is concave, the social planner (e.g., a public school) benefits more from leaving no one behind, making sure that all players (e.g., pupils) achieve a minimum level of output (e.g., basic level on a standardized test).

The next proposition shows that, despite pursuing a global objective over all outputs, i.e., $\sum_{i} W\left(x_{i}\right)$, the planner can focus her attention on one type of players only.

Proposition 6 (Tail-End Outputs). Suppose the value function is quadratic and $n_{A}=n_{B}$. Further, suppose the social planner maximizes $\sum_{i=1}^{n_{A}} W\left(x_{A, i}\right)+\sum_{i=1}^{n_{B}} W\left(x_{B, i}\right)$.

(i) If $W(x)$ is convex, the social planner should provide aggregate reference points from diverse reference groups in ahead-seeking environments and provide the full reference distributions from uniform reference groups in behind-averse environments.

(ii) If $W(x)$ is concave, the social planner should provide aggregate reference points from diverse reference groups in behind-averse environments and provide the full reference distributions from uniform reference groups in ahead-seeking environments.

The social planner will thus choose the reference structure in a way that maximizes the outputs of type- $A$ players when her payoff function is convex; and in a way that maximizes the outputs of type- $B$ players when her payoff function is concave (cf. Table 1). Effectively, the social planner can focus on maximizing the output of the best player $\left(\max \max _{i} x_{i}\right)$, i.e., on "developing a star," or on maximizing the output of the worst player $\left(\max \min _{i} x_{i}\right)$, i.e., on "leaving no one behind," without consideration of the other players' outputs depending on whether her objective function $W(x)$ is convex or concave.

As an illustration of Proposition 6, let us consider the social comparisons prevailing in elementary education in Asia and in the United States. The notorious competitiveness in Asian cultures is more consistent with the ahead-seeking behavior whereas Americans tend to be afraid of falling into the traps of "unhealthy competition," making children unduly anxious, consistent with the behind-averse behavior. ${ }^{11}$ The objective of the social planner (i.e., the school or the government) is however likely to be concave under either system given the early stage of elementary education. ${ }^{12}$

\footnotetext{
${ }^{11}$ Stevenson et al. (1990, p. 79) report that "having their child near the mean was sufficient for the American mothers to be satisfied; having a child above the mean was more often a requirement for mothers in Taipei and Sendai."

${ }^{12}$ In particular, Stevenson et al. (1990, p. 25) argue that in developed countries such as the U.S., China, and especially Japan, "the goal of [elementary] education is to reduce individual differences among children." By contrast, Duflo et al. (2011) argue that for such developing countries as Kenya, the teachers' objective function may be convex in the students' performance.
} 
Hence, the way a social planner should set the reference structure should be adapted to the mode of social comparison. Asian classes indeed tend to be streamed by "student quality," fostering greater group uniformity, and grades are usually fully revealed to all; whereas, U.S. classes are seldom streamed until much later, keeping them diverse, and grades are not typically publicized. These observations appear consistent with the recommendations of Proposition 6.

\section{Utility-Focused Strategies}

Instead of (or in addition to) caring about output, social planners could care about the players' well-being. In this section, we adopt the perspective of the players and assess their satisfaction under the different game settings. In contrast to the previous section, which demonstrated an alignment between the social planner's objective and the outputs of one type of players, maximizing player satisfaction is not without tensions.

We first study the impact of reference points on individual utilities. As shown in Lemma A-7 in Appendix A, type- $A$ players prefer diverse reference groups and type- $B$ players prefer uniform reference groups irrespective of whether they are behind-averse or ahead-seeking. Intuitively, type$A$ players prefer being part of a diverse group to affirm their superiority whereas type- $B$ players prefer being part of a uniform group to avoid being compared to players with greater innate ability.

The impact of reference point granularity on player utility is more ambiguous. Specifically, Lemma A-8 in Appendix A shows that when the value function is quadratic and $n_{A}=n / 2$, type$A$ players always prefer aggregate reference points. By contrast, type- $B$ players prefer aggregate reference points when they are behind-averse and full reference distributions otherwise.

Table 2 Reference structures that maximize the utilities of type- $\theta$ players when $n_{A}=n_{B}$ and when the value function is quadratic

\begin{tabular}{|c|c|c|}
\hline Objective & Ahead-seeking environment & Behind-averse environment \\
\hline \hline To increase $U_{A}:$ & diverse ref. group, aggregate ref. point & diverse ref. group, aggregate ref. point \\
\hline To increase $U_{B}:$ & uniform ref. group, full ref. distribution & uniform ref. group, aggregate ref. point \\
\hline
\end{tabular}

These results, summarized in Table 2, reveal that the preferences of type- $A$ and type- $B$ players are often misaligned. For example, a social planner seeking to please type- $A$ players in an aheadseeking environment would set aggregate reference points obtained from a diverse reference group, although type- $B$ players would prefer full reference distributions based on uniform reference groups.

Comparing Tables 1 and 2 reveals that the alignment between output maximization and utility maximization depends on the mode of social comparison at work. In an ahead-seeking environment, the reference structure that maximizes the output of a particular type of players is the same as the one that maximizes their utility. Consequently, this reference structure will allow one type of 
players to enjoy both high output and high utility, whereas the other type of players will achieve low performance and experience low utility. In this way, ahead-seeking behavior leads to favoritism.

By contrast when players are behind-averse, output maximization is not aligned with utility maximization. When maximizing the output of one particular type of players, the social planner may encounter dissatisfaction from that same type of players. Such environments may resemble boot camps, where high output is accompanied by poor satisfaction.

Instead of focusing on one particular type of players, the social planner could aim at maximizing the total utility. The following proposition shows that total utility is higher with diverse reference groups when players are ahead-seeking and with uniform reference groups otherwise.

Proposition 7 (Total Utility). Suppose that the value function is quadratic. Then, the social planner, maximizing total utility $\left(\sum_{i=1}^{n_{A}} U_{A, i}+\sum_{i=1}^{n_{B}} U_{B, i}\right)$, should use diverse reference groups in ahead-seeking environments and uniform reference groups in behind-averse environments; with these optimal reference groups, she is indifferent about the level of granularity of the reference point.

Is total utility maximization consistent with total output maximization? Comparing Propositions 5 and 7 reveals that reference group diversity affects total utility, but not total output, whereas reference point granularity affects total output, but not total utility (at least when reference groups are optimally chosen). Consequently, it is possible for the social planner to simultaneously maximize total output and total utility, by adopting the appropriate combination of reference point granularity and group diversity. Although "the best of both worlds" is a theoretical possibility, we caution that the social planner may end up with a reference structure that displeases all players in some way. For example, in ahead-seeking environments with a majority of type- $B$ players, the social planner could choose diverse reference groups (which type- $B$ players dislike) to maximize total utility and disclose individual performance (which type- $A$ players dislike) to maximize total output. Preferences are so misaligned that neither type of players is satisfied with the choice of reference structure, even though it jointly maximizes total utility and total output. Worse than not pleasing everyone, the social planner may thus end up not pleasing anyone.

Is total utility maximization consistent with the goals of a social planner who wishes to "develop stars" or "leave no one behind"? By comparing Propositions 6 and 7, we observe that maximizing total utility is aligned with the social planner's objective if it is convex but not when it is concave. It is indeed ironic that, when the social planner wants to leave no one behind, her most preferred reference structure may in fact generate the least total utility.

Finally, another important determinant of player satisfaction is equity. Customers do not like to be treated unfairly. How do we measure or even define equity? We do not attempt to answer this 
question in this paper. Instead, we restrict our discussion to two "equity principles," which provide precise guidelines for what we believe constitutes fair treatment of players.

\section{Equity Principle 1 Higher output should reflect greater effort.}

The first principle states that output should reflect the amount of effort put in. Since players differ in their intrinsic abilities, their outputs may not correspond to how hard they work. In our model, the output of type- $B$ players is identical to their effort; however, type- $A$ players need only to put in $x-k$ units of effort to get output $x$. This equity principle thus abstracts away from differences in ability and states that any output variation should reflect differences in effort.

Although the output of type- $A$ players is always (weakly) greater than the output of type- $B$ players in our model, it appears from the equilibrium conditions that type- $B$ players exert higher effort when players are behind-averse $\left(x_{B} \geq x_{A}-k\right)$ and that the opposite holds true when they are ahead-seeking $\left(x_{B, i} \leq x_{A, j}-k\right.$ for all $i=1, \ldots, n_{B}$ and $\left.j=1, \ldots, n_{A}\right)$. Hence, the first equity principle is satisfied in ahead-seeking environments and violated in behind-averse environments.

While the first principle above requires output to correspond to effort, our second principle of equity requires output to correspond to ability.

\section{Equity Principle 2 Higher output should reflect greater ability.}

The second principle states that type- $A$ players, given their greater innate ability, should get higher output. Moreover, among players of the same type, output should be the same. When all players share the same type, any output dispersion would indeed not reflect their common ability and could therefore be considered as artificial.

From Propositions 1-4, it appears that ahead-seeking behavior leads to an artificial dispersion of outputs since players of the same type end up having different outputs. However, behind-averse behavior ensures that all players of the same type have the same output. Hence, our second equity principle is satisfied in behind-averse environments and violated in ahead-seeking environments.

In sum, it will be impossible to simultaneously satisfy both equity principles. In addition to being unable to please everyone, a social planner will thus have to live with the facts that output may not reflect effort when players are behind-averse and that output may not reflect ability when players are ahead-seeking. ${ }^{13}$ The violation of these equity principles will be exacerbated when the social planner measures performance inaccurately, which we examine in the following section.

\footnotetext{
${ }^{13}$ Interestingly, Stevenson et al. (1990, p. 66) report that in elementary education, Asian mothers, who are arguably more ahead-seeking, tend to associate academic performance with effort whereas American mothers, who are arguably more behind-averse, tend to associate academic performance with ability. Hence, the mothers' beliefs about academic performance tend to be biased towards satisfying our two equity principles.
} 


\section{Noisy Performance Metrics}

We have assumed so far that the players' outputs were perfectly observable. However in practice, performance metrics may inaccurately reflect output. For instance in education, a score on the final exam may inaccurately reflect the amount of learning. In this section, we generalize our model to account for inaccurate performance metrics $y_{i}\left(x_{i}\right)$.

Let $y_{i}\left(x_{i}\right)$ denote the performance metric of player $i$ 's output. For simplicity, we consider additive noise; that is, $y_{i}\left(x_{i}\right)=x_{i}+\epsilon_{i}$, with $\epsilon_{i}$ being normally distributed with mean zero (i.e., $\mathbb{E}\left[\epsilon_{i}\right]=0$ ) and standard deviation $\sigma$, and independent of $\epsilon_{j}, j \neq i$. Let $\phi(x)$ be the probability density function of the standard normal distribution and $\Phi(x)$ be its cumulative distribution function. Because outputs are unobservable, social comparisons bear on the performance metrics.

Similar to $\S 3$, the social planner can set the reference point for social comparisons on the average performance of the group, $\overline{\mathbf{y}}(\mathbf{x}) \equiv \sum_{j} y_{j}\left(x_{j}\right) / n$, thereby creating an aggregate reference point. In that case, the utility functions for ahead-seeking or behind-averse players are respectively:

$$
U_{i}^{(\alpha a)}\left(x_{i} ; \mathbf{x}_{-i}\right)=V_{i}\left(x_{i}\right)+n \alpha \cdot \mathbb{E}\left[y_{i}\left(x_{i}\right)-\overline{\mathbf{y}}(\mathbf{x})\right]^{+} \text {and } U_{i}^{(\beta a)}\left(x_{i} ; \mathbf{x}_{-i}\right)=V_{i}\left(x_{i}\right)-n \beta \cdot \mathbb{E}\left[\overline{\mathbf{y}}(\mathbf{x})-y_{i}\left(x_{i}\right)\right]^{+} .
$$

Alternatively, the social planner can provide the full reference distribution so as to induce social comparisons on individual performances. In that case, the utility functions are, respectively for ahead-seeking and behind-averse players,

$U_{i}^{(\alpha f)}\left(x_{i} ; \mathbf{x}_{-i}\right)=V_{i}\left(x_{i}\right)+\alpha \sum_{j} \mathbb{E}\left[y_{i}\left(x_{i}\right)-y_{j}\left(x_{j}\right)\right]^{+}$and $U_{i}^{(\beta f)}\left(x_{i} ; \mathbf{x}_{-i}\right)=V_{i}\left(x_{i}\right)-\beta \sum_{j} \mathbb{E}\left[y_{j}\left(x_{j}\right)-y_{i}\left(x_{i}\right)\right]^{+}$.

When players are behind-averse, there is a unique equilibrium (see Proposition A-1 in Appendix A). Moreover when $\sigma \rightarrow 0$, the equilibrium coincides with the selected equilibrium in $\S \S 5-6 .{ }^{14}$

By contrast when players are ahead-seeking, establishing the existence of an equilibrium turns out to be more complicated because the game is submodular, for which little theory exists (Vives 1999). Existence of an equilibrium can be however guaranteed with an aggregate reference point since the game is in that case aggregative (Kukushkin 2004). Moreover, Proposition A-2 in Appendix A shows that (i) when $\sigma \rightarrow 0$, there exists a unique pure-strategy Nash equilibrium and that equilibrium is identical to the equilibria presented in Propositions 3 and 4; and, (ii) when $\sigma$ is large, there exists a Nash equilibrium in which all players of the same type choose the same output.

The model with noisy performance metrics thus naturally reduces to our base model presented in $\S 3$ when the noise $(\sigma)$ tends to zero. More interestingly, when the noise is very large, the effect of social comparison persists, both when players are behind-averse and when they are ahead-seeking,

\footnotetext{
${ }^{14}$ The noisy performance framework provides another justification for selecting that particular equilibrium in $\S \S 5-6$
} beyond risk dominance and stochastic stability. 
Figure 4 Equilibrium outputs when players are ahead-seeking and belong to a diverse reference group
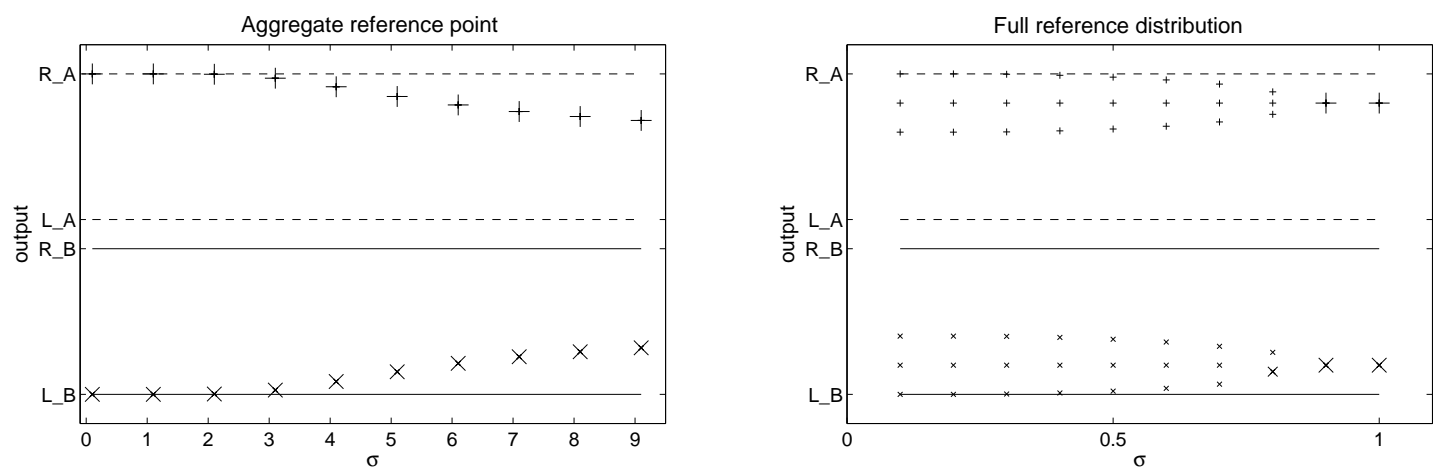

Note. The equilibria are reached through a sequential tâtonnement process, starting from $x_{\theta}=\left(V_{\theta}^{\prime}\right)^{-1}(-\alpha(n-1) / 2)$, $\theta=A, B$. The parameters are: $V_{B}(x)=100 x-x^{2}, V_{A}(x+k)=V_{B}(x), k=6, \alpha=2, n_{A}=n_{B}=3$. Type- $A$ and type- $B$ players are respectively represented with $a$ ' + ' and $a$ ' $X$ ' markers. The size of each marker is proportional to the number of players choosing that particular output.

in the sense that the equilibrium output exceeds the maximizer of $V_{\theta}(x)$. However, the dispersion in outputs among players of the same type completely vanishes when the performance metric is very noisy. Intuitively, the performance metric is so noisy that there is a high chance that any overinvestment in output will not translate into higher performance, therefore making such investment unattractive.

Figure 4 illustrates the evolution of the equilibrium outcomes with ahead-seeking players as $\sigma$ increases. For a range of small values of $\sigma$, the equilibrium outcomes are identical to the ones characterized in $\S 4$ for the case of accurate performance metrics. As $\sigma$ further increases, the equilibrium changes to gradually converge to the equilibrium characterized in Proposition A-2(ii) in Appendix A. Throughout that process, the structure of the equilibrium remains stable, the outputs being accumulated in a two-point distribution with an aggregate reference point and being more evenly spread within players of the same type with the full reference distribution.

With only one uniform reference group, some structural results can be obtained for the intermediate values of $\sigma$. (The proofs are omitted for brevity.) With an aggregate reference point, one can show the existence of an equilibrium when $n$ is even and $V(x)$ is quadratic such that $x_{1}=\ldots=x_{\frac{n}{2}}$ and $x_{\frac{n}{2}+1}=\ldots=x_{n}$. That is, we expect the two-point nature of the output distribution to be preserved, but the two points to get closer to each other as $\sigma$ increases. With the full reference distribution, one can show the existence of an equilibrium $\mathbf{x}^{*}$ when $n=3$ and $V(x)$ is quadratic, such that $x_{2}=\left(x_{1}+x_{3}\right) / 2$ when $\sigma V^{\prime \prime}(x) / \alpha \geq-0.3182$ and such that $x_{1}=x_{2} \leq x_{3}$ when $\sigma V^{\prime \prime}(x) / \alpha \leq-0.5658$. That is, we expect gradual bunching of outputs as $\sigma$ increases. Figure 5 illustrates the evolution of equilibrium outputs in a uniform reference group as $\sigma$ increases. 
Figure 5 Equilibrium outputs when players are ahead-seeking and belong to a uniform reference group
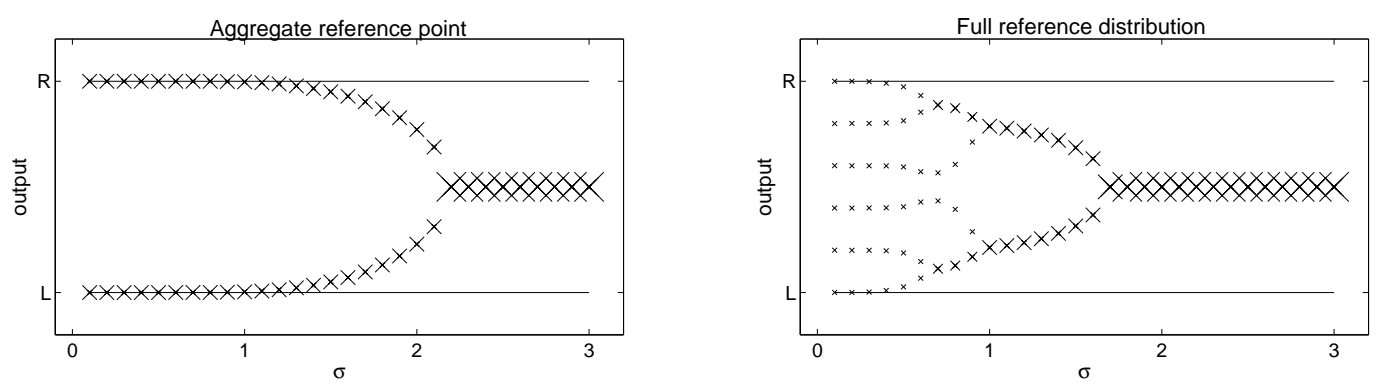

Note. The equilibria are reached through a sequential tâtonnement process, starting from $x=\left(V^{\prime}\right)^{-1}(-\alpha(n-1) / 2)$. The parameters are: $V(x)=100 x-x^{2}, \alpha=2, n=6$. The size of each marker is proportional to the number of players choosing that particular output.

\section{Conclusion}

In this paper, we study how social planners can manipulate reference points and reference groups to make full use of social comparison effects. We conclude that the optimal reference structure depends critically on the mode of social comparison effect at work: our recommendations for aheadseeking environments (where players derive pleasure from being ahead of others) and behind-averse environments (where players dislike being behind others) can be starkly different. Specifically, we find that the polarization effect induced by ahead-seeking behavior and the clustering effect induced by behind-averse behavior are the strongest when the planner uses diverse reference groups with an aggregate reference point, but can be mitigated with uniform reference groups and/or full reference distributions. Our analysis provides precise answers that depend on the social planner's objectives. However, we caution that player dissatisfaction often arises because it is seldom possible to please everyone or to treat everyone equitably.

Although considered fixed in our paper, the mode of social comparisons can sometimes be manipulated by the social planner. By publicly recognizing winners or losers, the social planner can accentuate the ahead-seeking or behind-averse character of the comparisons. The social planner may try to elicit ahead-seeking sentiments in high-performing players (so they strive to be even better) and behind-averse sentiments in low-performance players (so they try to keep up). In addition, the social planner can try to amplify the strength of social comparison effects, e.g., by adding injunctive messages (e.g., sad or smiley faces; see Schultz et al. 2007) to descriptive messages.

Social comparisons can be ordinal rather than cardinal. For example, a social planner could provide information about relative ranks, such as categorical feedback (e.g., course grades), without disclosing the absolute performances. With ordinal comparisons, the player game in our model may resemble a Bertrand game, potentially leading to no pure-strategy equilibrium or, in the words of Festinger (1954), no "state of social quiescence." 
Table A-1 Equilibrium first-order conditions.

\begin{tabular}{|c|c|c|c|}
\hline Environment & Ref. pt. granularity & Diverse group of size $n=n_{A}+n_{B}$ & Uniform group of size $n_{\theta}, \theta=A, B$ \\
\hline \multirow[t]{2}{*}{ Behind-averse } & Aggregate point & $\begin{array}{c}V_{A}^{\prime}\left(x_{A}^{(\beta a 2)}\right)=0 \\
V_{B}^{\prime}\left(x_{B}^{(\beta a 2)}\right)+\beta(n-1)=0\end{array}$ & $\begin{array}{l}V_{A}^{\prime}\left(x_{A}^{(\beta a 1)}\right)+\beta \frac{n_{A}-1}{2}=0 \\
V_{B}^{\prime}\left(x_{B}^{(\beta a 1)}\right)+\beta \frac{n_{B}-1}{2}=0\end{array}$ \\
\hline & Full distribution & $\begin{array}{c}V_{A}^{\prime}\left(x_{A}^{(\beta f 2)}\right)+\beta \frac{n_{A}-1}{2}=0 \\
V_{B}^{\prime}\left(x_{B}^{(\beta f 2)}\right)+\beta n_{A}+\beta \frac{n_{B}-1}{2}=0\end{array}$ & $\begin{array}{l}V_{A}^{\prime}\left(x_{A}^{(\beta f 1)}\right)+\beta \frac{n_{A}-1}{2}=0 \\
V_{B}^{\prime}\left(x_{B}^{(\beta f 1)}\right)+\beta \frac{n_{B}-1}{2}=0\end{array}$ \\
\hline \multirow[t]{2}{*}{ Ahead-seeking } & Aggregate point & $\begin{array}{c}V_{A}^{\prime}\left(x_{A}^{(\alpha a 2)}\right)+\alpha(n-1)=0 \\
V_{B}^{\prime}\left(x_{B}^{(\alpha a 2)}\right)=0\end{array}$ & $\begin{array}{l}V_{A}^{\prime}\left(x_{A, i}^{(\alpha a 1)}\right)+\alpha\left(n_{A}-1\right) \mathbb{1}_{\left[i>m_{A}\right]}=0 \\
V_{B}^{\prime}\left(x_{B, i}^{(\alpha a 1)}\right)+\alpha\left(n_{B}-1\right) \mathbb{1}_{\left[i>m_{B}\right]}=0\end{array}$ \\
\hline & Full distribution & $\begin{array}{c}V_{A}^{\prime}\left(x_{A, i}^{(\alpha f 2)}\right)+\alpha\left(n_{B}+i-1\right)=0 \\
V_{B}^{\prime}\left(x_{B, i}^{(\alpha f 2)}\right)+\alpha(i-1)=0\end{array}$ & $\begin{array}{l}V_{A}^{\prime}\left(x_{A, i}^{(\alpha f 1)}\right)+\alpha(i-1)=0 \\
V_{B}^{\prime}\left(x_{B, i}^{(\alpha f 1)}\right)+\alpha(i-1)=0\end{array}$ \\
\hline
\end{tabular}

Note: $m_{\theta}$ is such that $U_{\theta, i}^{(\alpha a 1)}\left(L_{\theta}^{\alpha} ; \overline{\mathbf{x}}_{-i}\right) \geq U_{\theta, i}^{(\alpha a 1)}\left(R_{\theta}^{\alpha} ; \overline{\mathbf{x}}_{-i}\right)$ when $\overline{\mathbf{x}}_{-i}=\left(\left(m_{\theta}-1\right) L_{\theta}+\left(n_{\theta}-m_{\theta}\right) R_{\theta}\right) /\left(n_{\theta}-1\right)$ and $U_{\theta, i}^{(\alpha a 1)}\left(R_{\theta}^{\alpha} ; \overline{\mathbf{x}}_{-i}\right) \geq$ $U_{\theta, i}^{(\alpha a 1)}\left(L_{\theta}^{\alpha} ; \overline{\mathbf{x}}_{-i}\right)$ when $\overline{\mathbf{x}}_{-i}=\left(m_{\theta} L_{\theta}+\left(n_{\theta}-m_{\theta}-1\right) R_{\theta}\right) /\left(n_{\theta}-1\right)$.

Finally, group performance can be enhanced through other social mechanisms, such as complementarities in production (e.g., classroom case discussion) and peer pressure (e.g., weight watcher programs). We hope that this paper will generate further research interest in these group dynamics.

\section{Appendix}

\section{A. Proofs and Supplementary Results}

Notations. We use the following notation for each of our eight game configurations, depending on whether players are ahead-seeking or behind-averse ( $\alpha$ or $\beta$ ), whether they receive an aggregate reference point or the full reference distribution ( $a$ or $f$ ), and whether their reference group is uniform or diverse, i.e., comprising 1 or 2 player types ( 1 or 2 ). For instance, $x_{A i}^{(\alpha f 1)}$ refers to the equilibrium strategy played by player $i$ of type $A$ when players are ahead-seeking, and have the full reference distribution from a uniform reference group, all players being of type $A$; and $U_{A i}^{(\alpha f 1)}$ refers to his utility. For $\xi=\alpha, \beta$ and $r=a, f$, we denote $\mathbf{x}^{[\xi r 2]}$ as the output of two diverse sections of size $n$, each consisting of $n / 2$ players of each type, and $\mathbf{x}^{[\xi r 1]}$ as the output of two uniform sections, with one section consisting of $n$ type- $A$ players and the other section consisting of $n$ type- $B$ players. Table A-1 provides a summary of the equilibrium conditions from Propositions 1-4.

Lemma A-1. Suppose players are behind-averse. Then, in any pure-strategy Nash equilibrium, all players of the same type must choose the same output.

Proof. Suppose there is a pure-strategy Nash equilibrium $\mathbf{x}^{*}$ such that $x_{i}^{*}<x_{j}^{*}$ with both $i, j$ of type $\theta$. Note that $\mathbf{x}_{-j}^{*} \leq \mathbf{x}_{-i}^{*}$ componentwise, so $U_{\theta}^{\prime}\left(x ; \mathbf{x}_{-j}^{*}\right) \leq U_{\theta}^{\prime}\left(x ; \mathbf{x}_{-i}^{*}\right)$ for all $x$. Thus, we must have $\arg \max U_{\theta}\left(x ; \mathbf{x}_{-j}^{*}\right) \leq \arg \max U_{\theta}\left(x ; \mathbf{x}_{-i}^{*}\right)$, which implies $x_{j}^{*} \leq x_{i}^{*}$, a contradiction.

Proof of Proposition 1. By Lemma A-1 in appendix, $x_{\theta, i}=x_{\theta}$ for all $i$. Given that $n\left[\overline{\mathbf{x}}-x_{i}\right]^{+}=\left[\sum_{j \neq i} x_{j}-\right.$ $\left.(n-1) x_{i}\right]^{+}=(n-1)\left[\overline{\mathbf{x}}_{-i}-x_{i}\right]^{+}$, the utility functions and first derivatives can be expressed as:

$$
U_{\theta}\left(x_{i} ; \mathbf{x}_{-i}\right)=V_{\theta}\left(x_{i}\right)-(n-1) \beta \cdot\left[\overline{\mathbf{x}}_{-i}-x_{i}\right]^{+} \text {and } U_{\theta}^{\prime}\left(x_{i} ; \mathbf{x}_{-i}\right)=V_{\theta}^{\prime}\left(x_{i}\right)+(n-1) \beta \cdot \mathbb{1}_{\left[\overline{\mathbf{x}}_{-i} \geq x_{i}\right]} .
$$


Given any $\mathbf{x}_{-i}, U_{\theta}^{\prime}\left(x_{i} ; \mathbf{x}_{-i}\right)$ is strictly decreasing in $x_{i}$, so $U_{\theta}$ is strictly concave and has a unique maximum.

(i) Suppose $n_{A} \geq 1$ and $n_{B} \geq 1$. In any equilibrium, we must have $x_{\theta} \in\left[L_{\theta}, R_{\theta}\right]$, and since $L_{A}>R_{B}$, it follows that $x_{A}>\overline{\mathbf{x}}_{-i}>x_{B}$. Using the first-order condition, we must have $V_{A}^{\prime}\left(x_{A}^{*}\right)=0$ and $V_{B}^{\prime}\left(x_{B}^{*}\right)+(n-1) \beta=0$, so $x_{A}^{*}=L_{A}$ and $x_{B}^{*}=R_{B}$.

(ii) Now, suppose all players are of type- $\theta$ (i.e., $n_{\theta}=n$ ). Any equilibrium must have $x_{\theta} \in\left[C_{\theta}^{\beta}(0), C_{\theta}^{\beta}(n-1)\right]$. This is indeed an equilibrium because for any small $\epsilon>0, U_{\theta}^{\prime}\left(x_{\theta}-\epsilon ; \mathbf{x}_{-i}\right)=V_{\theta}^{\prime}\left(x_{\theta}-\epsilon\right)+(n-1) \beta>V_{\theta}^{\prime}\left(x_{\theta}\right)+$ $(n-1) \beta \geq V_{\theta}^{\prime}\left(C_{\theta}^{\beta}(n-1)\right)+(n-1) \beta=0$ and similarly $U_{\theta}^{\prime}\left(x_{\theta}+\epsilon ; \mathbf{x}_{-i}\right)<0$, so $x_{\theta}$ is a best response.

Proof of Proposition 2. The utility functions and first derivatives can be expressed as:

$$
U_{\theta}\left(x_{i} ; \mathbf{x}_{-i}\right)=V_{\theta}\left(x_{i}\right)-\beta \sum_{j \neq i}\left[x_{j}-x_{i}\right]^{+} \text {and } U_{\theta}^{\prime}\left(x_{i} ; \mathbf{x}_{-i}\right)=V_{\theta}^{\prime}\left(x_{i}\right)+\beta \sum_{j \neq i} \mathbb{1}_{\left[x_{j} \geq x_{i}\right]} .
$$

Given any $\mathbf{x}_{-i}, U_{\theta}^{\prime}\left(x_{i} ; \mathbf{x}_{-i}\right)$ is strictly decreasing in $x_{i}$, so $U_{\theta}$ is strictly concave and has a unique maximum. Since $x_{\theta} \in\left[L_{\theta}, R_{\theta}\right]$ and $L_{A}>R_{B}$, we must have that $x_{A}>x_{B}$. Therefore, any type- $A$ player is behind between 0 and $n_{A}-1$ other players, and any type- $B$ player is behind between $n_{A}$ and $n-1$ other players. Thus, any equilibrium must have $x_{A} \in\left[C_{A}^{\beta}(0), C_{A}^{\beta}\left(n_{A}-1\right)\right]$ and $x_{B} \in\left[C_{B}^{\beta}\left(n_{A}\right), C_{B}^{\beta}(n-1)\right]$. It remains to show that such a strategy profile is indeed an equilibrium. For any small $\epsilon>0$, note that $U_{B}^{\prime}\left(x_{B}-\epsilon ; \mathbf{x}_{-i}\right)=$ $V_{B}^{\prime}\left(x_{B}-\epsilon\right)+(n-1) \beta>V_{B}^{\prime}\left(x_{B}\right)+(n-1) \beta \geq V_{B}^{\prime}\left(C_{B}^{\beta}(n-1)\right)+(n-1) \beta=0$ and similarly $U_{B}^{\prime}\left(x_{B}+\epsilon ; \mathbf{x}_{-i}\right)<0$, so $x_{B}$ is a best response. Similarly $x_{A}$ is a best response.

LEmmA A-2. Suppose players are ahead-seeking and have an aggregate reference point. Then, in any purestrategy Nash equilibrium, the output of each type- $\theta$ player must be either $L_{\theta}$ or $R_{\theta}$.

Proof. Depending on whether $x_{i}$ is larger or smaller than $\mathbf{x}_{-i}$, we know $U_{\theta}^{\prime}\left(x_{i} ; \overline{\mathbf{x}}_{-i}\right)$ is either $V_{\theta}^{\prime}\left(x_{i}\right)$ or $V_{\theta}^{\prime}\left(x_{i}\right)+(n-1) \alpha$. Therefore, the only choices that satisfy the first-order conditions are $L_{\theta}$ and $R_{\theta}$.

LEMmA A-3. Suppose players are ahead-seeking and have an aggregate reference point. There is a unique point $c_{\theta}=R_{\theta}-\frac{V_{\theta}\left(L_{\theta}\right)-V_{\theta}\left(R_{\theta}\right)}{(n-1) \alpha} \in\left[L_{\theta}, R_{\theta}\right]$ such that $U_{\theta}\left(L_{\theta} ; \mathbf{x}_{-i}\right) \geq U_{\theta}\left(R_{\theta} ; \mathbf{x}_{-i}\right)$ when $\overline{\mathbf{x}}_{-i} \geq c_{\theta}$ and $U_{\theta}\left(L_{\theta} ; \mathbf{x}_{-i}\right) \leq$ $U_{\theta}\left(R_{\theta} ; \mathbf{x}_{-i}\right)$ when $\overline{\mathbf{x}}_{-i} \leq c_{\theta}$.

Proof of Lemma A-3. Given that $n\left[x_{i}-\overline{\mathbf{x}}\right]^{+}=\left[(n-1) x_{i}-\sum_{j \neq i} x_{j}\right]^{+}=(n-1)\left[x_{i}-\overline{\mathbf{x}}_{-i}\right]^{+}$, the utility functions and first derivatives can be expressed as:

$$
U_{\theta}\left(x_{i} ; \mathbf{x}_{-i}\right)=V_{\theta}\left(x_{i}\right)+(n-1) \alpha \cdot\left[x_{i}-\overline{\mathbf{x}}_{-i}\right]^{+} \text {and } U_{\theta}^{\prime}\left(x_{i} ; \mathbf{x}_{-i}\right)=V_{\theta}^{\prime}\left(x_{i}\right)+(n-1) \alpha \cdot \mathbb{1}_{\left[x_{i} \geq \overline{\mathbf{x}}_{-i}\right]} .
$$

By Lemma A-2, each player chooses either $L_{\theta}$ or $R_{\theta}$. When $\overline{\mathbf{x}}_{-i} \leq L_{\theta}$, we have $U_{\theta}\left(L_{\theta} ; \mathbf{x}_{-i}\right) \leq U_{\theta}\left(R_{\theta} ; \mathbf{x}_{-i}\right)$ since $R_{\theta}$ maximizes utility for a player ahead of the mean. When $\overline{\mathbf{x}}_{-i} \geq R_{\theta}$, we have $U_{\theta}\left(L_{\theta} ; \mathbf{x}_{-i}\right) \geq U_{\theta}\left(R_{\theta} ; \mathbf{x}_{-i}\right)$ since $L_{\theta}$ maximizes utility for a player behind the mean. As $\overline{\mathbf{x}}_{-i}$ increases from $L_{\theta}$ to $R_{\theta}, U_{\theta}\left(L_{\theta} ; \mathbf{x}_{-i}\right)$ remains constant and equal to $V_{\theta}\left(L_{\theta}\right)$ whereas $U_{\theta}\left(R_{\theta} ; \mathbf{x}_{-i}\right)$ decreases. Finally, we obtain the expression for $c_{\theta}$ using $U_{\theta}\left(L_{\theta} ; \mathbf{x}_{-i}\right)=U_{\theta}\left(R_{\theta} ; \mathbf{x}_{-i}\right)$ when $\overline{\mathbf{x}}_{-i}=c_{\theta}$, i.e., $V_{\theta}\left(L_{\theta}\right)=V_{\theta}\left(R_{\theta}\right)+(n-1) \alpha \cdot\left(R_{\theta}-c_{\theta}\right)$.

Proof of Proposition 3. The proof uses Lemmas A-2 and A-3 in the appendix. Define $l=\frac{(n-1) c_{B}+L_{B}}{n}$ and $r=\frac{(n-1) c_{A}+R_{A}}{n}$, where $c_{A}, c_{B}$ are as defined in Lemma A-3. Consider case (ii) of what we need to prove, so we have $\frac{n_{A} R_{A}+n_{B} L_{B}}{n}<r$, which implies $\frac{\left(n_{A}-1\right) R_{A}+n_{B} R_{B}}{n-1}<c_{A}$. Suppose we have an equilibrium in which player $i$ of type- $A$ chooses $L_{A}$. Since the largest choices of all other players are $R_{\theta}$, we have $\overline{\mathbf{x}}_{-i} \leq \frac{\left(n_{A}-1\right) R_{A}+n_{B} R_{B}}{n-1}<c_{A}$, 
which by Lemma A-3 implies that the best response of player $i$ is $R_{A}$ instead, so we have a contradiction. In other words, all type- $A$ players must choose $R_{A}$ in equilibrium. Similarly, all type- $B$ players must choose $L_{B}$ in equilibrium. Thus, we have a unique equilibrium with $m_{A}=0$ and $m_{B}=n_{B}$.

Next, consider case (i). Since $\frac{n_{A} R_{A}+n_{B} L_{B}}{n}<l<r$, the same argument as the one above shows that all type$A$ players must choose $R_{A}$ in equilibrium. Further, $\frac{n_{A} R_{A}+n_{B} L_{B}}{n}<l$ implies $\frac{n_{A} R_{A}+\left(n_{B}-1\right) L_{B}}{n-1}<c_{B}$. Thus, there is a unique $m_{B} \in\left[1, n_{B}-1\right]$ such that $\frac{n_{A} R_{A}+\left(m_{B}-1\right) L_{B}+\left(n_{B}-m_{B}\right) R_{B}}{n-1}>c_{B}$ and $\frac{n_{A} R_{A}+m_{B} L_{B}+\left(n_{B}-m_{B}-1\right) R_{B}}{n-1}<c_{B}$. By Lemma A-3, both these inequalities must be satisfied if it is an equilibrium for exactly $m_{B}$ type- $B$ players to choose $L_{B}$. Therefore, we have a unique equilibrium with $m_{B}$ as defined above. The proof for case (iii) is similar.

LEmmA A-4. Suppose players are ahead-seeking and have the full reference distribution. Then, in any pure-strategy Nash equilibrium, no pair of players choose the same output.

Proof. Suppose there is a Nash equilibrium $\mathbf{x}^{*}$ in which player $i$ (of type $\theta$ ) chooses $x_{i}=z, k \geq 1$ other players also choose $z$, and exactly $k^{\prime}$ players choose output strictly less than $z$. Then, for any small $\epsilon>0, U_{\theta}^{\prime}(z-\epsilon ; \mathbf{x})=V_{\theta}^{\prime}(z-\epsilon)+k^{\prime} \alpha$ and $U_{\theta}^{\prime}\left(z+\epsilon ; \mathbf{x}_{-i}\right)=V_{\theta}^{\prime}(z+\epsilon)+\left(k+k^{\prime}\right) \alpha$. Since $k \geq 1$, we must have $U_{\theta}^{\prime}\left(z-\epsilon ; \mathbf{x}_{-i}\right)<U_{\theta}^{\prime}\left(z+\epsilon ; \mathbf{x}_{-i}\right)$. Thus, $x_{i}=z$ cannot be a best response and we have a contradiction.

Proof of Proposition 4. The utility functions and first derivatives can be expressed as:

$$
U_{\theta}\left(x_{i} ; \mathbf{x}_{-i}\right)=V_{\theta}\left(x_{i}\right)+\alpha \sum_{j \neq i}\left[x_{i}-x_{j}\right]^{+} \text {and } U_{\theta}^{\prime}\left(x_{i} ; \mathbf{x}_{-i}\right)=V_{\theta}^{\prime}\left(x_{i}\right)+\alpha \sum_{j \neq i} \mathbb{1}_{\left[x_{i} \geq x_{j}\right]}
$$

By Lemma A-4, all output choices are distinct. Since $L_{A}>R_{B}$, the lowest $n_{B}$ outputs come from type- $B$ players. By the first-order condition, the $i$ th lowest output must satisfy $V_{B}^{\prime}\left(x_{i}\right)+(i-1) \alpha=0$, leading to the same vector $\mathbf{x}^{*}$ as in the proposition. It remains to verify that $\mathbf{x}^{*}$ is indeed an equilibrium. Consider player $i$ of type $\theta$ who chooses $x_{\theta, i}^{*}$ that is greater than or equal to exactly $\tilde{n}_{i}$ elements of $\mathbf{x}^{*}$. Based on the first-order condition for $x_{\theta, i}^{*}$, we have $U_{\theta}^{\prime}\left(x_{i} ; \mathbf{x}_{-i}^{*}\right)=V_{\theta}^{\prime}\left(x_{i}\right)+\tilde{n}_{i} \alpha=0$ at $x_{i}=x_{\theta, i}^{*}$ and $U_{\theta}^{\prime}\left(x_{i} ; \mathbf{x}_{-i}^{*}\right)>0$ for $x_{i} \in\left[x_{\theta, i-1}^{*}, x_{\theta, i}^{*}\right)$. Next, based on the first-order condition for $x_{\theta, i-1}^{*}$, we have $U_{\theta}^{\prime}\left(x_{i} ; \mathbf{x}_{-i}^{*}\right)>0$ for $x_{i} \in\left[x_{\theta, i-2}^{*}, x_{\theta, i-1}^{*}\right)$. Continuing in this way, we have $U_{\theta}^{\prime}\left(x_{i} ; \mathbf{x}_{-i}^{*}\right)>0$ for every $x_{i} \in\left[L_{\theta}, x_{\theta, i}^{*}\right)$. Similarly, we can show $U_{\theta}^{\prime}\left(x_{i} ; \mathbf{x}_{-i}^{*}\right)<0$ for every $x_{i} \in\left(x_{\theta, i}^{*}, R_{\theta}\right]$. Therefore, $x_{\theta, i}^{*}$ is a best response and we have a Nash equilibrium.

LEMmA A-5. $\quad$ 1. $x_{A, i}^{[\alpha f 2]} \geq x_{A, i}^{[\alpha f 1]}$ and $x_{B, i}^{[\alpha f 2]} \leq x_{B, i}^{[\alpha f 1]} ; x_{A, i}^{[\alpha a 2]} \geq x_{A, i}^{[\alpha a 1]}$ and $x_{B, i}^{[\alpha a 2]} \leq x_{B, i}^{[\alpha a 1]}$; 2. $x_{A}^{[\beta f 2]} \leq x_{A}^{[\beta f 1]}$ and $x_{B}^{[\beta f 2]} \geq x_{B}^{[\beta f 1]} ; x_{A}^{[\beta a 2]} \leq x_{A}^{[\beta a 1]}$ and $x_{B}^{[\beta a 2]} \geq x_{B}^{[\beta a 1]}$.

Proof. The proof follows from comparing the respective entries in Table A-1 after making the following substitutions for all $\xi=\alpha, \beta, r=a, f$, and $i=1, \ldots, 2 n$ : (i) $x_{\theta, i}^{[\xi r 2]}=x_{\theta,\left\lceil\frac{i}{2}\right\rceil}^{(\xi r 2)}$ with $n_{A}=n / 2$ and $n_{B}=n / 2$ and (ii) $x_{\theta, i}^{[\xi r 1]}=x_{\theta, i}^{(\xi r 1)}$ with $n_{A}=n$ and $n_{B}=n$. For any $i=1, \ldots, 2 n$ :

1. $V_{A}^{\prime}\left(x_{A, i}^{[\alpha f 1]}\right)+\alpha(i-1)=0=V_{A}^{\prime}\left(x_{A, i}^{[\alpha f 2]}\right)+\alpha\left(n / 2+\left\lceil\frac{i}{2}\right\rceil-1\right) \geq V_{A}^{\prime}\left(x_{A, i}^{[\alpha f 2]}\right)+\alpha(i-1)$. Hence, $x_{A, i}^{[\alpha f 1]} \leq x_{A, i}^{[\alpha f 2]}$. And, $V_{B}^{\prime}\left(x_{B, i}^{[\alpha f 2]}\right)+\alpha\left(\left\lceil\frac{i}{2}\right\rceil-1\right)=0=V_{B}^{\prime}\left(x_{B, i}^{[\alpha f 1]}\right)+\alpha(i-1) \geq V_{B}^{\prime}\left(x_{B, i}^{[\alpha f 1]}\right)+\alpha\left(\left\lceil\frac{i}{2}\right\rceil-1\right)$. Hence, $x_{B, i}^{[\alpha f 2]} \leq x_{B, i}^{[\alpha f 1]}$. And, $V_{A}^{\prime}\left(x_{A, i}^{[\alpha a 1]}\right)+\alpha(n-1) \mathbb{1}_{\left[i>m_{A}\right]}=0=V_{A}^{\prime}\left(x_{A}^{[\alpha a 2]}\right)+\alpha(n-1) \geq V_{A}^{\prime}\left(x_{A}^{[\alpha a 2]}\right)+\alpha(n-1) \mathbb{1}_{\left[i>m_{A}\right]}$. Hence, $x_{A, i}^{[\alpha a 1]} \leq x_{A, i}^{[\alpha a 2]}$. And, $V_{B}^{\prime}\left(x_{B}^{[\alpha a 2]}\right)=0=V_{B}^{\prime}\left(x_{B, i}^{[\alpha a 1]}\right)+\alpha(n-1) \mathbb{1}_{\left[i>m_{B}\right]} \geq V_{B}^{\prime}\left(x_{B, i}^{[\alpha a 1]}\right)$. Hence, $x_{B, i}^{[\alpha a 2]} \leq x_{B, i}^{[\alpha a 1]}$. 
2. $V_{A}^{\prime}\left(x_{A}^{[\beta f 2]}\right)+\beta \frac{n / 2-1}{2}=0=V_{A}^{\prime}\left(x_{A}^{[\beta f 1]}\right)+\beta \frac{n-1}{2} \geq V_{A}^{\prime}\left(x_{A}^{[\beta f 1]}\right)+\beta \frac{n / 2-1}{2}$. Hence, $x_{A}^{[\beta f 2]} \leq x_{A}^{[\beta f 1]}$. And, $V_{B}^{\prime}\left(x_{B}^{[\beta f 1]}\right)+\beta \frac{n-1}{2}=0=V_{B}^{\prime}\left(x_{B}^{[\beta f 2]}\right)+\beta n / 2+\beta \frac{n / 2-1}{2} \geq V_{B}^{\prime}\left(x_{B}^{[\beta f 2]}\right)+\beta \frac{n-1}{2}$. Hence, $x_{B}^{[\beta f 1]} \leq x_{B}^{[\beta f 2]}$. And, $V_{A}^{\prime}\left(x_{A}^{[\beta a 2]}\right)=0=V_{A}^{\prime}\left(x_{A}^{[\beta a 1]}\right)+\beta \frac{n-1}{2} \geq V_{A}^{\prime}\left(x_{A}^{[\beta a 1]}\right)$. Hence, $x_{A}^{[\beta a 2]} \leq x_{A}^{[\beta a 1]}$. And, $V_{B}^{\prime}\left(x_{B}^{[\beta a 1]}\right)+\beta \frac{n-1}{2}=0=$ $V_{B}^{\prime}\left(x_{B}^{[\beta a 2]}\right)+\beta(n-1) \geq V_{B}^{\prime}\left(x_{B}^{[\beta a 2]}\right)+\beta \frac{n-1}{2}$. Hence, $x_{B}^{[\beta a 1]} \leq x_{B}^{[\beta a 2]}$.

Lemma A-6. $\quad$ 1. $x_{A, i}^{(\alpha a 2)} \geq x_{A, i}^{(\alpha f 2)}$ and $x_{B, i}^{(\alpha a 2)} \leq x_{B, i}^{(\alpha f 2)}$;

2. $x_{A, i}^{(\beta a 2)} \leq x_{A, i}^{(\beta f 2)}$ and $x_{B, i}^{(\beta a 2)} \geq x_{B, i}^{(\beta f 2)}$.

Proof. From Table A-1, we obtain:

1. For $i=1, \ldots, n_{A}, V_{A}^{\prime}\left(x_{A, i}^{(\alpha f 2)}\right)+\alpha\left(n_{B}+i-1\right)=0=V_{A}^{\prime}\left(x_{A}^{(\alpha a 2)}\right)+\alpha(n-1) \geq V_{A}^{\prime}\left(x_{A}^{(\alpha a 2)}\right)+\alpha\left(n_{B}+i-1\right)$; i.e., $x_{A, i}^{(\alpha f 2)} \leq x_{A}^{(\alpha a 2)}$. For $i=1, \ldots, n_{B}, V_{B}^{\prime}\left(x_{B}^{(\alpha a 2)}\right)=0=V_{B}^{\prime}\left(x_{B, i}^{(\alpha f 2)}\right)+\alpha(i-1) \geq V_{B}^{\prime}\left(x_{B, i}^{(\alpha f 2)}\right)$; i.e., $x_{B, i}^{(\alpha a 2)} \leq x_{B}^{(\alpha f 2)}$.

2. For $i=1, \ldots, n_{A}, V_{A}^{\prime}\left(x_{A}^{(\beta a 2)}\right)=0=V_{A}^{\prime}\left(x_{A}^{(\beta f 2)}\right)+\beta \frac{n_{A}-1}{2} \geq V_{A}^{\prime}\left(x_{A}^{(\beta f 2)}\right)$; i.e., $x_{A}^{(\beta a 2)} \leq x_{A}^{(\beta f 2)}$. For $i=$ $1, \ldots, n_{B}, V_{B}^{\prime}\left(x_{B}^{(\beta f 2)}\right)+\beta n_{A}+\beta \frac{n_{B}-1}{2}=0=V_{B}^{\prime}\left(x_{B}^{(\beta a 2)}\right)+\beta(n-1) \geq V_{B}^{\prime}\left(x_{B}^{(\beta a 2)}\right)+\beta n_{A}+\beta \frac{n_{B}-1}{2}$; i.e., $x_{B}^{(\beta f 2)} \leq$ $x_{B}^{(\beta a 2)}$.

Proof of Proposition 5. In Lemma EC.1 in the electronic companion, we show that $\sum_{i=1}^{n_{A}} x_{A, i}^{(\alpha a 2)}+$ $\sum_{i=1}^{n_{B}} x_{B, i}^{(\alpha a 2)} \leq \sum_{i=1}^{n_{A}} x_{A, i}^{(\alpha f 2)}+\sum_{i=1}^{n_{B}} x_{B, i}^{(\alpha f 2)}$ and $\sum_{i=1}^{n_{A}} x_{A, i}^{(\beta a 2)}+\sum_{i=1}^{n_{B}} x_{B, i}^{(\beta a 2)} \geq \sum_{i=1}^{n_{A}} x_{A, i}^{(\beta f 2)}+\sum_{i=1}^{n_{B}} x_{B, i}^{(\beta f 2)}$ if and only if $n_{A} \leq n_{B}$. And, that $\sum_{i=1}^{2 n} x_{i}^{[\alpha a 2]}=\sum_{i=1}^{2 n} x_{i}^{[\alpha a 1]}=\sum_{i=1}^{2 n} x_{i}^{[\alpha f 2]}=\sum_{i=1}^{2 n} x_{i}^{[\alpha f 1]}$ and $\sum_{i=1}^{2 n} x_{i}^{[\beta a 2]}=$ $\sum_{i=1}^{2 n} x_{i}^{[\beta a 1]}=\sum_{i=1}^{2 n} x_{i}^{[\beta f 2]}=\sum_{i=1}^{2 n} x_{i}^{[\beta f 1]}$.

Proof of Proposition 6. When $n_{A}=n_{B}, \sum_{i=1}^{n_{A}} x_{A, i}^{(\xi r 2)}+\sum_{i=1}^{n_{B}} x_{B, i}^{(\xi r 2)}$ is constant across all $r=a, f$ by Proposition 5, for any $\xi=\alpha, \beta$. Note that $\mathbf{x}^{(\xi r 2)}=\left(\mathbf{x}_{B}^{(\xi r 2)}, \mathbf{x}_{A}^{(\xi r 2)}\right)$ is sorted in ascending order for any $\xi=\alpha, \beta$, and $r=a, f$. For all $\xi=\alpha, \beta$ and $r=a, f$, define $s_{l}^{(\xi r 2)}=\sum_{i=1}^{l} x_{i}^{(\xi r 2)}, l=1, \ldots, n$. In Lemma EC.2 in the electronic companion, we show that $s_{l}^{(\alpha a 2)} \leq s_{l}^{(\alpha f 2)}$ and $s_{l}^{(\beta f 2)} \leq s_{l}^{(\beta a 2)}$ for all $l \leq$ $n$. Hence, the sorted output vectors $\mathbf{x}^{(\xi r 2)}$ can be ordered according to majorization (Hardy et al. 1952, p. 45): $-\mathbf{x}^{(\alpha a 2)} \succ-\mathbf{x}^{(\alpha f 2)}$ and $-\mathbf{x}^{(\beta f 2)} \succ-\mathbf{x}^{(\beta a 2)}$. From Theorem 108 in Hardy et al. (1952), we obtain that $\sum_{i=1}^{n_{A}} W\left(x_{A, i}^{(\alpha a 2)}\right)+\sum_{i=1}^{n_{B}} W\left(x_{B, i}^{(\alpha a 2)}\right) \geq \sum_{i=1}^{n_{A}} W\left(x_{A, i}^{(\alpha f 2)}\right)+\sum_{i=1}^{n_{B}} W\left(x_{B, i}^{(\alpha f 2)}\right)$ and $\sum_{i=1}^{n_{A}} W\left(x_{A, i}^{(\beta f 2)}\right)+$ $\sum_{i=1}^{n_{B}} W\left(x_{B, i}^{(\beta f 2)}\right) \geq \sum_{i=1}^{n_{A}} W\left(x_{A, i}^{(\beta a 2)}\right)+\sum_{i=1}^{n_{B}} W\left(x_{B, i}^{(\beta a 2)}\right)$ if and only if $W(x)$ is convex.

Fix $\xi=\alpha, \beta$ and $r=a, f$. By Proposition $5, \sum_{i=1}^{2 n} x_{i}^{[\xi r 1]}=\sum_{i=1}^{2 n} x_{i}^{[\xi r 2]}$. Note that $\mathbf{x}^{[\xi r 1]}=\left(\mathbf{x}_{B}^{[\xi r 1]}, \mathbf{x}_{A}^{[\xi r 1]}\right)$ is sorted in ascending order, and so is $\mathbf{x}^{[\xi r 2]}$. Let us define $s_{l}^{(\xi r 1)}=\sum_{i=1}^{l} x_{i}^{[\xi r 1]}, l=1, \ldots, 2 n$. In Lemma EC.3 in the electronic companion, we show that $s_{l}^{(\beta r 1)} \leq \sum_{i=1}^{l} x_{i}^{[\beta r 2]}$ and $s_{l}^{(\alpha r 1)} \geq \sum_{i=1}^{l} x_{i}^{[\alpha r 2]}$ for all $l \leq$ $2 n$, for any $r=a, f$. Therefore, $-\mathbf{x}^{[\alpha r 1]} \prec-\mathbf{x}^{[\alpha r 2]}$ and $-\mathbf{x}^{[\beta r 1]} \succ-\mathbf{x}^{[\beta r 2]}$, for $r=a, f$. From Theorem 108 in Hardy et al. (1952), we then obtain that $\sum_{i=1}^{2 n} W\left(x_{i}^{[\alpha f 2]}\right) \geq \sum_{i=1}^{2 n} W\left(x_{i}^{[\alpha f 1]}\right), \sum_{i=1}^{2 n} W\left(x_{i}^{[\alpha a 2]}\right) \geq$ $\sum_{i=1}^{2 n} W\left(x_{i}^{[\alpha a 1]}\right), \sum_{i=1}^{2 n} W\left(x_{i}^{[\beta f 2]}\right) \leq \sum_{i=1}^{2 n} W\left(x_{i}^{[\beta f 1]}\right)$, and $\sum_{i=1}^{2 n} W\left(x_{i}^{[\beta a 2]}\right) \leq \sum_{i=1}^{2 n} W\left(x_{i}^{[\beta a 1]}\right)$ if and only if $W(x)$ is convex.

Lemma A-7. 1. When $V(x)$ is quadratic, $U_{A, i}^{[\alpha f 2]} \geq U_{A, i}^{[\alpha f 1]}$ and $U_{B, i}^{[\alpha f 2]} \leq U_{B, i}^{[\alpha f 1]}$; When $V(x)$ is quadratic, $U_{A, i}^{[\alpha a 2]} \geq U_{A, i}^{[\alpha a 1]}$ and $U_{B, i}^{[\alpha a 2]} \leq U_{B, i}^{[\alpha a 1]}$;

2. $U_{A}^{[\beta f 2]} \geq U_{A}^{[\beta f 1]}$ and $U_{B}^{[\beta f 2]} \leq U_{B}^{[\beta f 1]} ; U_{A}^{[\beta a 2]} \geq U_{A}^{[\beta a 1]}$ and $U_{B}^{[\beta a 2]} \leq U_{B}^{[\beta a 1]}$.

Proof. The first result follows from Lemma EC.4 in the electronic companion.

Fix $r=a, f$. Because $x_{A, i}^{[\beta r 2]}=x_{A}^{[\beta r 2]}$ and $x_{A, i}^{[\beta r 1]}=x_{A}^{[\beta r 1]}$ for all $i, U_{A}^{[\beta r 2]}=V_{A}\left(x_{A}^{[\beta r 2]}\right)$ and $U_{A}^{[\beta r 1]}=V_{A}\left(x_{A}^{[\beta r 1]}\right)$. Because $x_{A}^{[\beta r 2]} \leq x_{A}^{[\beta r 1]}$ by Lemma A-5, $U_{A}^{[\beta r 2]} \geq U_{A}^{[\beta r 1]}$ since $V_{A}^{\prime}(x) \leq 0$ for any $x \geq L_{A}$. 
We have $U_{B}^{[\beta f 2]}=V_{B}\left(x_{B}^{[\beta f 2]}\right)-\beta n_{A}\left[x_{A}^{[\beta f 2]}-x_{B}^{[\beta f 2]}\right]^{+} \leq V_{B}\left(x_{B}^{[\beta f 2]}\right)$ and $U_{B, i}^{[\beta a 2]}=V_{B}\left(x_{B}^{[\beta a 2]}\right)-\beta(n-$ 1) $\left[\overline{\mathbf{x}}_{-i}^{[\beta a 2]}-x_{B}^{[\beta a 2]}\right]^{+} \leq V_{B}\left(x_{B}^{[\beta a 2]}\right)$, whereas $U_{B}^{[\beta f 1]}=V_{B}\left(x_{B}^{[\beta f 1]}\right)$ and $U_{B}^{[\beta a 1]}=V_{B}\left(x_{B}^{[\beta a 1]}\right)$. Fix $r=a, f$. Because $x_{B}^{[\beta r 2]} \geq x_{B}^{[\beta r 1]}$ by Lemma A-5, $V_{B}\left(x_{B}^{[\beta r 2]}\right) \leq V_{B}\left(x_{B}^{[\beta r 1]}\right)$ by concavity. Hence, $U_{B}^{[\beta r 1]} \geq U_{B}^{[\beta r 2]}$.

LEMmA A-8. $\quad$ 1. $U_{B, i}^{(\alpha a 2)} \leq U_{B, i}^{(\alpha f 2)}$ for all $i$; when $V(x)$ is quadratic, $U_{A, i}^{(\alpha a 2)} \geq U_{A, i}^{(\alpha f 2)}$ for all $i$ if $n_{A} \leq n / 2$; 2. $U_{A, i}^{(\beta a 2)} \geq U_{A, i}^{(\beta f 2)}$ for all $i$; when $V(x)$ is quadratic, $U_{B, i}^{(\beta a 2)} \geq U_{B, i}^{(\beta f 2)}$ for all $i$ if $n_{A} \geq n / 2$.

Proof. From Table A-1, $x_{B}^{(\alpha a 2)}=L_{B}^{\alpha}$; therefore, $U_{B}^{(\alpha a 2)}=V\left(L_{B}^{\alpha}\right)$. On the other hand, $U_{B, i}^{(\alpha f 2)}=$ $V\left(x_{B, i}^{(\alpha f 2)}\right)+\alpha \sum_{j \neq i}\left[x_{B, i}^{(\alpha f 2)}-x_{B, j}^{(\alpha f 2)}\right]^{+} \geq V\left(L_{B}^{\alpha}\right)$ given that $L_{B}^{\alpha}$ is feasible. Hence, $U_{B, i}^{(\alpha f 2)} \geq U_{B, i}^{(\alpha a 2)}$ for all $i$.

From Table A-1, $x_{A}^{(\beta a 2)}=L_{A}^{\beta} \geq x_{B}^{(\beta a 2)}$; hence, $U_{A}^{(\beta a 2)}=V\left(L_{A}^{\beta}\right)$. Since $L_{A}^{\beta}$ maximizes $V(x), U_{A}^{(\beta a 2)} \geq U_{A}^{(\beta f 2)}$.

The remaining two results follow from Lemma EC.5 in the electronic companion.

Proof of Proposition 7. Lemma EC.6 in the electronic companion shows that $\sum_{i=1}^{n_{A}} U_{A, i}^{[\alpha f 2]}+\sum_{i=1}^{n_{B}} U_{B, i}^{[\alpha f 2]}=$ $\sum_{i=1}^{n_{A}} U_{A, i}^{[\alpha a 2]}+\sum_{i=1}^{n_{B}} U_{B, i}^{[\alpha a 2]} \geq \sum_{i=1}^{n_{A}} U_{A, i}^{[\alpha f 1]}+\sum_{i=1}^{n_{B}} U_{B, i}^{[\alpha f 1]}=\sum_{i=1}^{n_{A}} U_{A, i}^{[\alpha a 1]}+\sum_{i=1}^{n_{B}} U_{B, i}^{[\alpha a 1]}$ and $\sum_{i=1}^{n_{A}} U_{A, i}^{[\beta a 1]}+$ $\sum_{i=1}^{n_{B}} U_{B, i}^{[\beta a 1]}=\sum_{i=1}^{n_{A}} U_{A, i}^{[\beta f 1]}+\sum_{i=1}^{n_{B}} U_{B, i}^{[\beta f 1]} \geq \sum_{i=1}^{n_{A}} U_{A, i}^{[\beta a 2]}+\sum_{i=1}^{n_{B}} U_{B, i}^{[\beta a 2]} \geq \sum_{i=1}^{n_{A}} U_{A, i}^{[\beta f 2]}+\sum_{i=1}^{n_{B}} U_{B, i}^{[\beta f 2]}$.

Proposition A-1. With behind-averse players and noisy performance metrics, there exists a unique purestrategy Nash equilibrium $\mathrm{x}^{*}$ such that $x_{\theta, i}^{*}=x_{\theta}^{*}$ for $i=1, \ldots, n_{\theta}, \theta=A, B$, in which $\left(x_{B}^{*}, x_{A}^{*}\right)$ solve

(i) $V_{\theta}^{\prime}\left(x_{\theta}^{*}\right)+\beta(n-1) \Phi\left(\frac{n_{-\theta}\left(x_{-\theta}^{*}-x_{\theta}^{*}\right)}{\sigma \sqrt{n(n-1)}}\right)=0$ for $\theta=A, B$ with an aggregate reference point, and

(ii) $V_{\theta}^{\prime}\left(x_{\theta}^{*}\right)+\beta \frac{\left(n_{\theta}-1\right)}{2}+\beta n_{-\theta} \Phi\left(\frac{x_{-\theta}^{*}-x_{\theta}^{*}}{\sigma \sqrt{2}}\right)=0$ for $\theta=A, B$ with the full reference distribution.

Proof. In both cases, existence is guaranteed given that $U_{i}\left(x_{i} ; \mathbf{x}_{-i}\right)$ is strictly concave for all $i$ (Fudenberg and Tirole 2000, Theorem 1.2). The game between all players of the same type is symmetric since they have the same utility functions and same action spaces. Moreover, the action spaces are completely ordered (since they are intervals) and the utility functions are strictly supermodular since $\frac{\partial^{2} U_{i}^{(\beta a)}\left(x_{i} ; \mathbf{x}_{-i}\right)}{\partial x_{j} \partial x_{i}}=\beta(n-1) \phi\left(\frac{x_{i}-\overline{\mathbf{x}}_{-i}}{\sqrt{\frac{n}{n-1}} \sigma}\right) \frac{1}{\sqrt{n(n-1)} \sigma}>0$ and $\frac{\partial^{2} U_{i}^{(\beta f)}\left(x_{i} ; \mathbf{x}_{-i}\right)}{\partial x_{j} \partial x_{i}}=\beta \phi\left(\frac{x_{i}-x_{j}}{\sqrt{2} \sigma}\right) \frac{1}{\sqrt{2} \sigma}>0$.

As a result for any $\mathbf{x}_{-\theta}$, the equilibrium between all type- $\theta$ players must be unique (Vives 1999). Therefore, $x_{\theta, i}=x_{\theta}$ for all $i=1, \ldots, n_{\theta}$. This yields the equilibrium conditions.

Uniqueness follows from the global univalence theorem (Gale and Nikaidô 1965) by showing that the Jacobian matrix obtained from the equilibrium conditions is diagonally-dominant:

$$
\begin{aligned}
& \left|\frac{\partial^{2} U_{\theta, i}^{(\beta a)}\left(x_{i} ; \mathbf{x}_{-i}\right)}{\partial x_{i}^{2}}\right|=-V_{\theta}^{\prime \prime}\left(x_{i}\right)+\beta \frac{(n-1)}{\sqrt{\frac{n}{n-1}} \sigma} \phi\left(\frac{x_{i}-\overline{\mathbf{x}}_{-i}^{*}}{\sqrt{\frac{n}{n-1}} \sigma}\right)>\sum_{j \neq i}\left|\frac{\partial^{2} U_{\theta, i}^{(\beta a)}\left(x_{i} ; \mathbf{x}_{-i}\right)}{\partial x_{j} \partial x_{i}}\right|, \\
& \left|\frac{\partial^{2} U_{\theta, i}^{(\beta f)}\left(x_{i} ; \mathbf{x}_{-i}\right)}{\partial x_{i}^{2}}\right|=-V_{\theta}^{\prime \prime}\left(x_{i}\right)+\beta \sum_{j \neq i} \phi\left(\frac{x_{i}-x_{j}^{*}}{\sqrt{2} \sigma}\right) \frac{1}{\sqrt{2} \sigma}>\sum_{j \neq i}\left|\frac{\partial^{2} U_{\theta, i}^{(\beta f)}\left(x_{i} ; \mathbf{x}_{-i}\right)}{\partial x_{j} \partial x_{i}}\right| .
\end{aligned}
$$

Proposition A-2. With ahead-seeking players and noisy performance metrics,

(i) When $\sigma \rightarrow 0$, there exists a unique pure-strategy Nash equilibrium that is identical to the equilibria presented in Propositions 3 and 4 .

(ii) When $\sigma \geq-\frac{\phi(0) \alpha(n-1)}{\max _{x} V^{\prime \prime}(x) \sqrt{\frac{n}{n-1}}}$, there exists a pure-strategy Nash equilibrium $\mathbf{x}^{*}$ such that $x_{\theta, i}^{*}=x_{\theta}^{*}$ for $i=1, \ldots, n_{\theta}, \theta=A, B$, in which $\left(x_{B}^{*}, x_{A}^{*}\right)$ solve

(ii.a) $V_{\theta}^{\prime}\left(x_{\theta}^{*}\right)+\alpha(n-1) \Phi\left(\frac{n_{-\theta}\left(x_{\theta}^{*}-x_{-\theta}^{*}\right)}{\sigma \sqrt{n(n-1)}}\right)=0$ for $\theta=A, B$ with an aggregate reference point, and

(ii.b) $V_{\theta}^{\prime}\left(x_{\theta}^{*}\right)+\alpha \frac{\left(n_{\theta}-1\right)}{2}+\alpha n_{-\theta} \Phi\left(\frac{x_{\theta}^{*}-x_{-\theta}^{*}}{\sqrt{2} \sigma}\right)=0$ for $\theta=A, B$, with the full reference distribution. 
Proof. (i) Consider first the case with the full reference distribution. Similar to Lemma A-4, one can show that $x_{i} \neq x_{j}$ in every equilibrium. Moreover, we obtain by L'Hospital's rule that $\lim _{\sigma \rightarrow 0} \phi\left(\frac{x_{i}-x_{j}}{\sqrt{2} \sigma}\right) \frac{1}{\sigma}=0$ for any $x_{i} \neq x_{j}$, i.e., $\lim _{\sigma \rightarrow 0} U_{\theta, i}^{\prime \prime}\left(x_{i} ; \mathbf{x}_{-i}\right)=V_{\theta}^{\prime \prime}\left(x_{i}\right)<0$. The first-order condition then yield $V_{\theta}^{\prime}\left(x_{j}\right)+\alpha(j-1)=0$ for all $j$. The rest of the proof then consists in showing, similar to the proof of Proposition 4, that setting $x_{i} \in\left[x_{j}, x_{j+1}\right]$ is never a best response exploiting the fact $U_{i}\left(x_{i} ; \mathbf{x}_{-i}\right)$ is strictly concave on that interval. The proof of the case with an aggregate reference point follows a similar argument.

(ii) Consider the case with an aggregate reference point. For any $x_{i}, \mathbf{x}_{-i}$,

$$
U_{\theta, i}^{\prime \prime}\left(x_{i} ; \mathbf{x}_{-i}\right)=V_{\theta}^{\prime \prime}(x)+\alpha(n-1) \phi\left(\frac{x_{i}-\overline{\mathbf{x}}_{-i}}{\sqrt{\frac{n}{n-1}} \sigma}\right) \frac{1}{\sqrt{\frac{n}{n-1}} \sigma} \leq \max _{x} V_{\theta}^{\prime \prime}(x)+\alpha(n-1) \phi(0) \frac{1}{\sqrt{\frac{n}{n-1}} \sigma} \leq 0
$$

by assumption on $\sigma$. Accordingly, $U_{\theta, i}\left(x ; \mathbf{x}_{-i}\right)$ is concave everywhere. As a result, there exists a pure-strategy Nash equilibrium (Fudenberg and Tirole 2000, Theorem 1.2). The argument for the case with the full reference distribution is identical. The first-order optimality conditions are, respectively with an aggregate reference point and the full reference distribution: $V_{\theta}^{\prime}\left(x_{i}^{*}\right)+\alpha(n-1) \Phi\left(\frac{x_{i}^{*}-\overline{\mathbf{x}}_{-i}}{\sqrt{\frac{n}{n-1} \sigma}}\right)=0$ and $V_{\theta}^{\prime}\left(x_{i}^{*}\right)+\alpha \sum_{j \neq i} \Phi\left(\frac{x_{i}^{*}-x_{j}^{*}}{\sqrt{2} \sigma}\right)=$ 0 . Setting $x_{\theta, i}^{*}=x_{\theta}^{*}$ for $i=1, \ldots, n_{\theta}$ in those equilibrium conditions yields the result.

\section{B. Model Extensions}

\section{B.1. Reference groups with both ahead-seeking and behind-averse players}

In this section, we characterize the equilibrium when the reference group consists of both ahead-seeking and behind-averse players. An illustration is provided in Appendix B.3.1. Let us respectively denote with $n_{\theta}^{\alpha}$ and $n_{\theta}^{\beta}$ the number of ahead-seeking and behind-averse type- $\theta$ players, with $n_{\theta}^{\alpha}+n_{\theta}^{\beta}=n_{\theta}$. Denote output as $\mathbf{x}=\left(\mathbf{x}_{B}^{\alpha}, \mathbf{x}_{B}^{\beta}, \mathbf{x}_{A}^{\alpha}, \mathbf{x}_{A}^{\beta}\right)$. Suppose that $n>n_{A}^{\beta}, n_{B}^{\beta}$ for otherwise, the setting would be identical to Propositions $1,2,3$, or 4 . As before, we assume non-overlapping strategy sets across types, i.e., $\max \left\{R_{B}^{\alpha}, R_{B}^{\beta}\right\}<L_{A}$.

Because Lemma A-1 and Lemmas A-2-A-4 continue to hold, the equilibrium here with an aggregate reference point superimposes the equilibria obtained in Propositions 1 and 3.

Proposition B-1. Suppose players have an aggregate reference point. There is a unique pure-strategy Nash equilibrium, in which all $n_{\theta}^{\beta}$ type- $\theta$ behind-averse players choose $z_{\theta}, m_{\theta}^{\alpha}$ type- $\theta$ ahead-seeking players choose $L_{\theta}^{\alpha}$ and $n_{\theta}^{\alpha}-m_{\theta}^{\alpha}$ of the remaining type- $\theta$ ahead-seeking players choose $R_{\theta}^{\alpha}$, for $\theta=A, B$. There exist constants $l \in\left[L_{B}^{\alpha}, R_{B}^{\alpha}\right]$ and $r \in\left[L_{A}^{\alpha}, R_{B}^{\alpha}\right]$ (so $L_{B}^{\alpha}<l<R_{B}^{\alpha}<L_{A}^{\alpha}<r<R_{A}^{\alpha}$ ) such that:

(i) If $\frac{n_{A}^{\alpha} R_{A}^{\alpha}+n_{A}^{\beta} L_{A}^{\beta}+n_{B}^{\beta} R_{B}^{\beta}+n_{B}^{\alpha} L_{B}^{\alpha}}{n} \in\left[L_{B}^{\alpha}, l\right)$, then $m_{A}^{\alpha}=0, \quad z_{A}=L_{A}^{\alpha}, \quad z_{B}=$ $\min \left\{R_{B}^{\beta}, \frac{m_{B}^{\alpha} L_{B}^{\alpha}+\left(n_{B}^{\alpha}-m_{B}^{\alpha}\right) R_{B}^{\alpha}+n_{A}^{\beta} L_{A}^{\beta}+n_{A}^{\alpha} R_{A}^{\alpha}}{n-n_{B}^{\beta}}\right\}$, and $m_{B}^{\alpha} \in\left[1, n_{B}^{\alpha}-1\right]$.

(ii) If $\frac{n_{A}^{\alpha} R_{A}^{\alpha}+n_{A}^{\beta} L_{A}^{\beta}+n_{B}^{\beta} R_{B}^{\beta}+n_{B}^{\alpha} L_{B}^{\alpha}}{n} \in(l, r)$, then $m_{A}^{\alpha}=0, z_{A}=L_{A}^{\alpha}, z_{B}=R_{B}^{\beta}$, and $m_{B}^{\alpha}=n_{B}^{\alpha}$.

(iii) $\frac{n_{A}^{\alpha} R_{A}^{\alpha}+n_{A}^{\beta} L_{A}^{\beta}+n_{B}^{\beta} R_{B}^{\beta}+n_{B}^{\alpha} L_{B}^{\alpha}}{n} \in\left(r, R_{A}^{\alpha}\right]$, then $m_{A}^{\alpha} \in\left[1, n_{A}^{\alpha}-1\right], z_{A}=\min \left\{R_{A}^{\beta}, \frac{n_{B}^{\alpha} L_{B}^{\alpha}+n_{B}^{\beta} R_{B}^{\beta}+m_{A}^{\alpha} L_{A}^{\alpha}+\left(n_{A}^{\alpha}-m_{A}^{\alpha}\right) R_{A}^{\alpha}}{n-n_{A}^{\beta}}\right\}$, $z_{B}=R_{B}^{\beta}$, and $m_{B}^{\alpha}=n_{B}^{\alpha}$.

Hence, the introduction of ahead-seeking players in the reference group makes behind-averse players stabilize on one equilibrium, unlike Proposition 2, which established existence of multiple equilibria.

Similarly, the equilibrium with both ahead-seeking and behind-averse players with the full reference distribution superimposes the equilibria obtained in Propositions 2 and 4. 
Proposition B-2. Suppose players have the full reference distribution. For any $m_{B}^{\alpha} \in\left\{0, \ldots, n_{B}^{\alpha}\right\}, m_{A}^{\alpha} \in$ $\left\{0, \ldots, n_{A}^{\alpha}\right\}, z_{B} \in\left[\max \left\{C_{B}^{\beta}\left(n_{B}^{\alpha}-m_{B}^{\alpha}+n_{A}\right), C_{B}^{\alpha}\left(m_{B}^{\alpha}-1\right)\right\}, \min \left\{C_{B}^{\beta}\left(n-1-m_{B}^{\alpha}\right), C_{B}^{\alpha}\left(m_{B}^{\alpha}+n_{B}^{\beta}\right)\right\}\right]$, and $z_{A} \in$ $\left[\max \left\{C_{A}^{\beta}\left(n_{A}^{\alpha}-m_{A}^{\alpha}\right), C_{A}^{\alpha}\left(n_{B}+m_{A}^{\alpha}-1\right)\right\}, \min \left\{C_{A}^{\beta}\left(n_{A}-m_{A}^{\alpha}-1\right), C_{A}^{\alpha}\left(n_{B}+m_{A}^{\alpha}+n_{A}^{\beta}\right)\right], \mathbf{x}^{*}\right.$ is a Nash equilibrium, defined as $x_{i}^{*}=C_{B}^{\alpha}(i-1)$ for $i=1, \ldots, m_{B}^{\alpha}, x_{i}^{*}=C_{B}^{\alpha}\left(n_{B}^{\beta}+i-1\right)$ for $i=m_{B}^{\alpha}+1, \ldots, n_{B}^{\alpha}, x_{i}^{*}=z_{B}$ for $i=n_{B}^{\alpha}+$ $1, \ldots, n_{B}, x_{i}^{*}=C_{A}^{\alpha}(i-1) \alpha$ for $i=n_{B}+1, \ldots, n_{B}+m_{A}^{\alpha}, x_{i}^{*}=C_{A}^{\alpha}\left(n_{A}^{\beta}+i-1\right)$ for $i=n_{B}+m_{A}^{\alpha}+1, \ldots, n_{B}+n_{A}^{\alpha}$, and $x_{i}^{*}=z_{A}$ for $i=n_{B}+n_{A}^{\alpha}+1, \ldots, n$.

It can be checked that a Nash equilibrium always exists since the value of $z_{B}$ that satisfies $C_{B}^{\beta}\left(n_{B}^{\alpha}-m_{B}^{\alpha}+\right.$ $\left.n_{A}\right)=C_{B}^{\alpha}\left(m_{B}^{\alpha}-1\right)$ is smaller than the allowed upper bound, $\min \left\{C_{B}^{\beta}\left(n-1-m_{B}^{\alpha}\right), C_{B}^{\alpha}\left(m_{B}^{\alpha}+n_{B}^{\beta}\right)\right\}$ when $n_{B}^{\beta} \geq 1$, and similarly for $z_{A}$. Moreover, the set of possible Nash equilibria is convex since the lower and upper bounds on $z_{\theta}$ are respectively convex and concave functions of $m_{\theta}^{\alpha}$, for $\theta=A, B$.

\section{B.2. Overlapping Strategy Sets}

In this appendix, we consider overlapping strategy sets, i.e., we allow for the difference between the two player types to be so small that $L_{A}<R_{B}$. The next four propositions list the equilibrium results, analogous to Propositions 1 to 4. Illustrations are provided in Appendix B.3.

We first consider behind-averse players. With overlapping strategy sets, it is now possible for all players to choose the same output in equilibrium, in contrast to our basic analysis, in which the players' choices cluster at one end of the strategy set (i.e., at the low end for type- $A$ and at the high end for type- $B$ players), but they do not coincide. Now, the choices of both types converge to the same point, which as before is nearer the upper end of type- $B$ 's strategy set and nearer the lower end of type- $A$ 's strategy set.

Proposition B-3. Suppose players are behind-averse and have an aggregate reference point. For any $z^{*} \in\left[L_{A}, R_{B}\right], x_{A}^{*}=x_{B}^{*}=z^{*}$ is a Nash equilibrium.

Proposition B-4. Suppose players are behind-averse and have the full reference distribution.

(i) For any $z^{*} \in\left[L_{A}, R_{B}\right], x_{A}^{*}=x_{B}^{*}=z^{*}$ is a Nash equilibrium.

(ii) For any $z_{A} \in\left[C_{A}^{\beta}(0), C_{A}^{\beta}\left(n_{A}-1\right)\right]$ and $z_{B} \in\left[C_{B}^{\beta}\left(n_{A}\right), C_{B}^{\beta}(n-1)\right]$ such that $z_{A}>z_{B}, x_{A}^{*}=z_{A}, x_{B}^{*}=z_{B}$ is a Nash equilibrium.

We next consider ahead-seeking players. We show that the equilibria have the same structure as in our basic analysis with non-overlapping strategy sets. With aggregate reference points, choices are polarized at either the lowest or the highest end of each type's strategy set. With the full reference distribution, choices are spread out along the strategy set such that no pair chooses the same output. These observations are incredibly robust. However, with overlapping strategy sets, there is a multitude of equilibria, in contrast to our basic analysis of non-overlapping strategy sets, which had a unique Nash equilibrium.

Proposition B-5. Suppose players are ahead-seeking and have an aggregate reference point. Consider a strategy profile $\mathbf{x}$ in which $m_{\theta}$ type- $\theta$ players choose $L_{\theta}$ and $n_{\theta}-m_{\theta}$ of the remaining type- $\theta$ players choose $R_{\theta}$, for $\theta=A, B$. Let $\overline{\mathbf{x}}$ be the average choice of all players. There exists constants $l, l^{\prime} \in\left[L_{B}, R_{B}\right]$ satisfying $L_{B}<l^{\prime}<l<R_{B}$ and $r, r^{\prime} \in\left[L_{A}, R_{A}\right]$ satisfying $L_{A}<r<r^{\prime}<R_{A}$ such that:

(i) If $l^{\prime}<\overline{\mathbf{x}}<l<r$ and $m_{A}=0$, then $\mathbf{x}$ is a Nash equilibrium. 
(ii) If $l<\overline{\mathbf{x}}<r$ and $m_{A}=0$ and $m_{B}=n_{B}$, then $\mathbf{x}$ is a Nash equilibrium.

(iii) If $l<r<\overline{\mathbf{x}}<r^{\prime}$ and $m_{B}=n_{B}$, then $\mathbf{x}$ is a Nash equilibrium.

(iv) If $l^{\prime}<\overline{\mathbf{x}}<r<l$ and $m_{A}=0$, then $\mathbf{x}$ is a Nash equilibrium.

(v) If $r<\overline{\mathbf{x}}<l$, then $\mathbf{x}$ is a Nash equilibrium.

(vi) If $r<l<\overline{\mathbf{x}}<r^{\prime}$ and $m_{B}=n_{B}$, then $\mathbf{x}$ is a Nash equilibrium.

Proposition B-6. Suppose players are ahead-seeking and have the full reference distribution. Consider a strategy profile $\mathbf{x}$ in which no two players of the same type choose the same output. Further, suppose $V_{A}^{\prime}\left(x_{A, i}\right)+\alpha \rho_{A, i}=0$ for $i=1, \ldots, n_{A}$, and $V_{B}^{\prime}\left(x_{B, i}\right)+\alpha \rho_{B, i}=0$ for $i=1, \ldots, n_{B}$, where $\rho_{\theta, i}$ is the number of players choosing a smaller output than the $i$-th type- $\theta$ player. Then $\mathbf{x}$ is a Nash equilibrium.

\section{B.3. Numerical Examples}

In this section, we present a series of numerical examples. In all cases, the equilibria are reached through a sequential tâtonnement process, starting from $x_{\theta}=L_{\theta}, \theta=A, B$. In each figure, type- $A$ and type- $B$ players are respectively represented with a ' + ' and a ' $x$ ' markers. The size of each marker is proportional to the number of players choosing that particular output. The dotted curves show the bounds $L_{A}, R_{A}$ within which outputs of type- $A$ players must lie, and the solid curves show analogous bounds $L_{B}, R_{B}$ for type- $B$ players. Ahead-seeking and behind-averse players are respectively displayed in grey and black. Ahead-seeking players tend to choose outputs near the outside boundaries $L_{B}^{\alpha}$ and $R_{A}^{\alpha}$ and behind-averse players tend to choose outputs near the inside boundaries $R_{B}^{\alpha}$ and $L_{A}^{\alpha}$.

B.3.1. Heterogeneous Groups and Overlapping Strategy Sets. We first present numerical examples of equilibrium outputs when (i) the reference group consist of both ahead-seeking and behind-averse players and (ii) strategy sets of type- $A$ and type- $B$ players overlap, thereby stress-testing our results in Propositions 1-4 to those assumptions and illustrating the analytical developments from Appendices B.1-B.2.

Figures B-1 and B-2 consider the case with individual references points whereas Figures B-3 and B-4 consider the cases with aggregate reference points. The parameters are: $V_{B}(x)=100 x-x^{2}, V_{A}(x+k)=V_{B}(x)$, $n_{A}=n_{B}=6$. Moreover, $\alpha=3$ and $\beta=2$ in Figures B-1 and B-3 and $\alpha=2$ and $\beta=3$ in Figures B-2 and B-4. We moreover consider asymmetric configurations of reference groups with $n_{A}^{\alpha}=n_{B}^{\alpha}=2$ ahead-seeking players in the left figures and $n_{A}^{\alpha}=n_{B}^{\alpha}=4$ ahead-seeking players in the right figures.

Overall, all figures exhibit clustering behavior of behind-averse players and dispersion of ahead-seeking players, either very polarized at the outer boundaries of the strategy sets (aggregate reference point), or more uniformly distributed, but still anchored at the outer boundaries of the strategy sets (full reference distribution). Overall, the insights obtained in Propositions 1-4, with non-overlapping strategy sets and common kind of social comparison at work, appear to be very robust to those assumptions.

B.3.2. Value Function and Overlapping Strategy Sets. We finally display in Figure B-5 the equilibrium outputs when $V(x)=100 x-x^{p}$ for various values of $p$. The purpose of this study is to show that the equilibrium outcomes obtained with a quadratic value function $(p=2)$ are representative of the outcomes obtained with other value functions, and therefore, that the results that have been obtained for the quadratic case are robust (Propositions 5-7 and Lemmas A-7 and A-8 which lie behind Table 2). A byproduct of this 
Figure B-1 Equilibrium outputs with the full reference distribution and diverse reference groups, consisting of both ahead-seeking and behind-averse players with $\alpha=3$ and $\beta=2$.
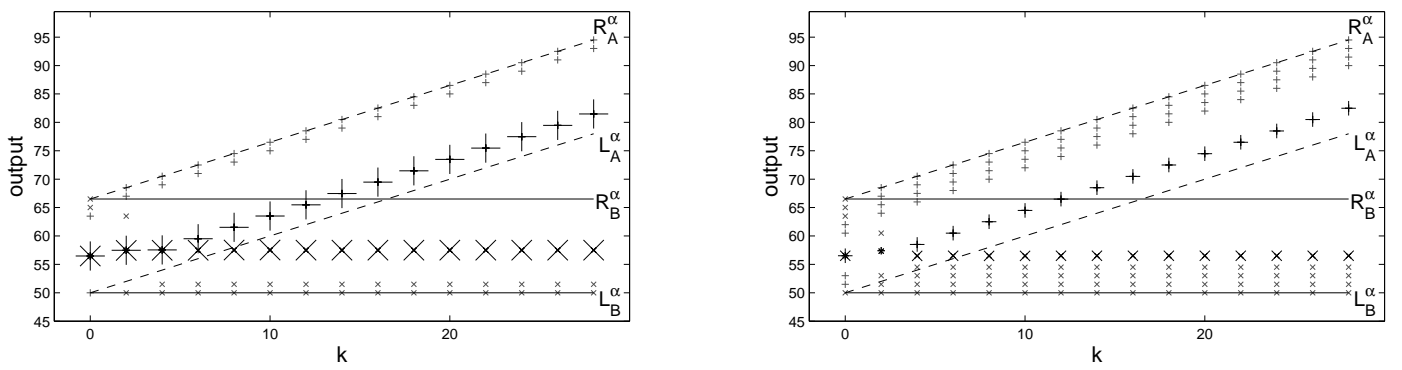

Note. $n_{A}^{\alpha}=n_{B}^{\alpha}=2$ (left) and $n_{A}^{\alpha}=n_{B}^{\alpha}=4$ (right).

Figure B-2 Equilibrium outputs with the full reference distribution and diverse reference groups, consisting of both ahead-seeking and behind-averse players with $\alpha=2$ and $\beta=3$.
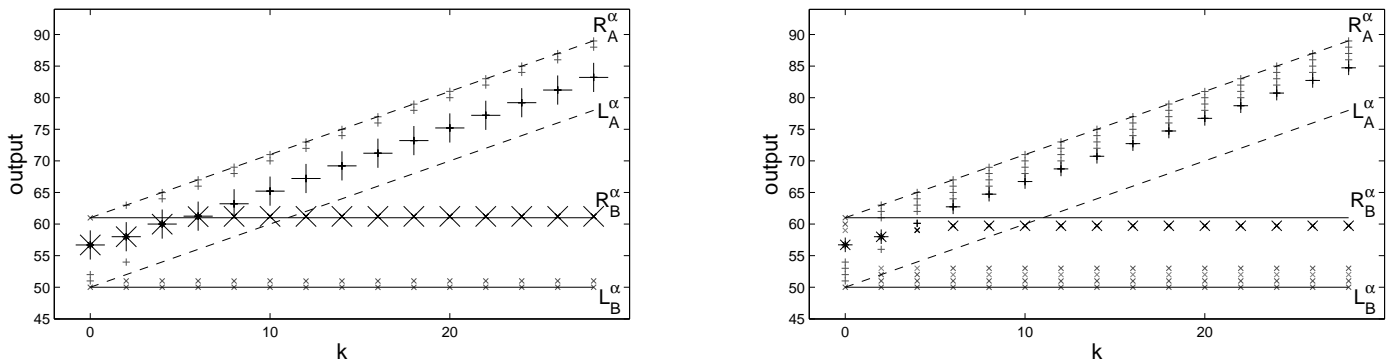

Note. $n_{A}^{\alpha}=n_{B}^{\alpha}=2$ (left) and $n_{A}^{\alpha}=n_{B}^{\alpha}=4$ (right).

Figure B-3 Equilibrium outputs with an aggregate reference point and diverse reference groups, consisting of both ahead-seeking and behind-averse players with $\alpha=3$ and $\beta=2$.
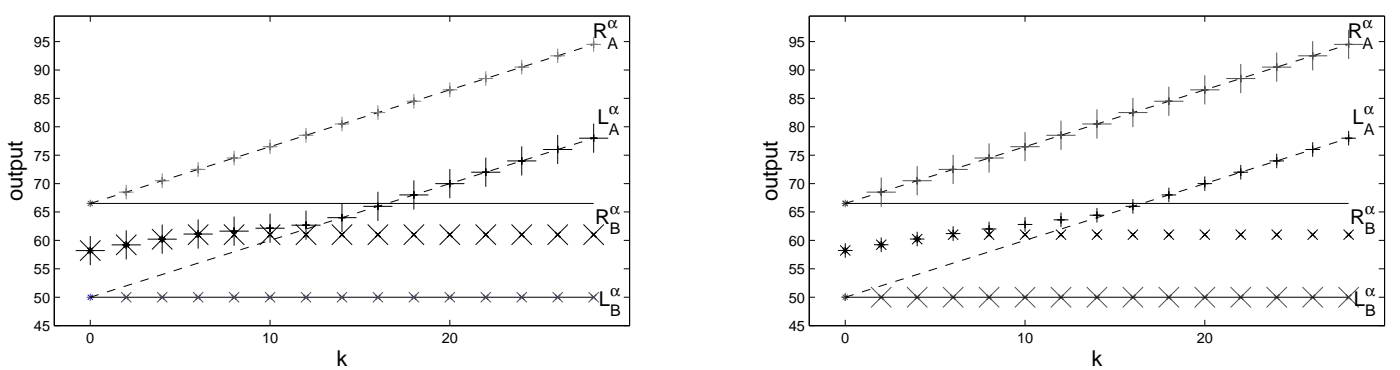

Note. $n_{A}^{\alpha}=n_{B}^{\alpha}=2$ (left) and $n_{A}^{\alpha}=n_{B}^{\alpha}=4$ (right).

numerical analysis is to demonstrate the robustness of Propositions 1-4 with respect to the assumption that the strategy sets do not overlap, similar to Appendix B.2.

Because the equilibrium outputs may vary widely (e.g., $\left[L_{B}, R_{B}\right]=[11.7,12.5]$ and $\left[L_{A}, R_{A}\right]=[17.7,18.5]$ when $p=2.5$ and $\left[L_{B}, R_{B}\right]=[4444,5378]$ and $\left[L_{A}, R_{A}\right]=[4450,5384]$ when $\left.p=1.5\right)$, we linearly normalize all quantities between $L_{B}$ and $R_{A}$ for comparison purposes. Note that the action sets $\left[L_{B}, R_{B}\right]$ and $\left[L_{A}, R_{A}\right]$ are overlapping when $p \leq 1.75$ and become clustered near $L_{B}$ and $R_{A}$ as $p$ increases. 
Figure B-4 Equilibrium outputs with an aggregate reference point and diverse reference groups, consisting of both ahead-seeking and behind-averse players with $\alpha=2$ and $\beta=3$.
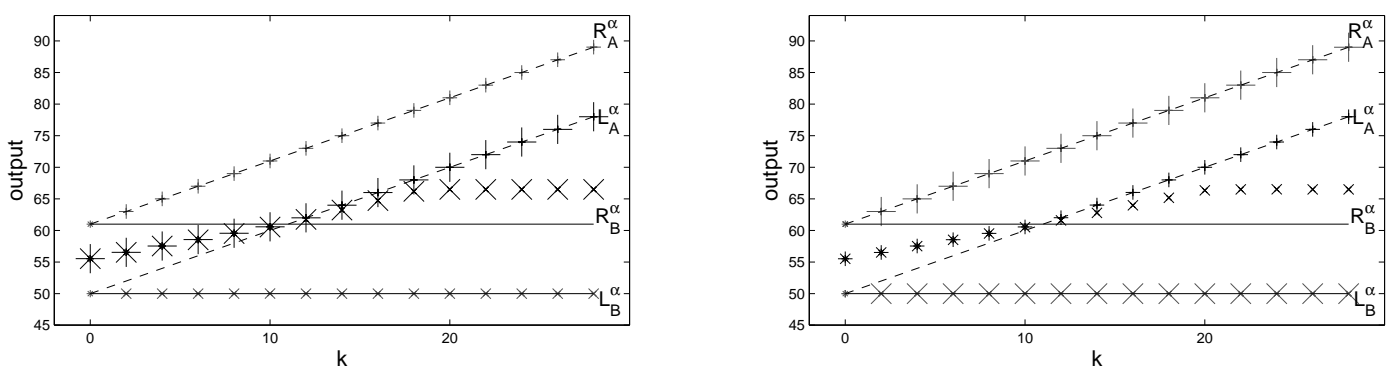

Note. $n_{A}^{\alpha}=n_{B}^{\alpha}=2$ (left) and $n_{A}^{\alpha}=n_{B}^{\alpha}=4$ (right).

Figure B-5 Equilibrium outputs $\mathbf{x}^{*}$ for different value functions $V(x)=100 x-x^{p}$.

Ahead-seeking, full reference distribution

Ahead-seeking, aggregate reference point
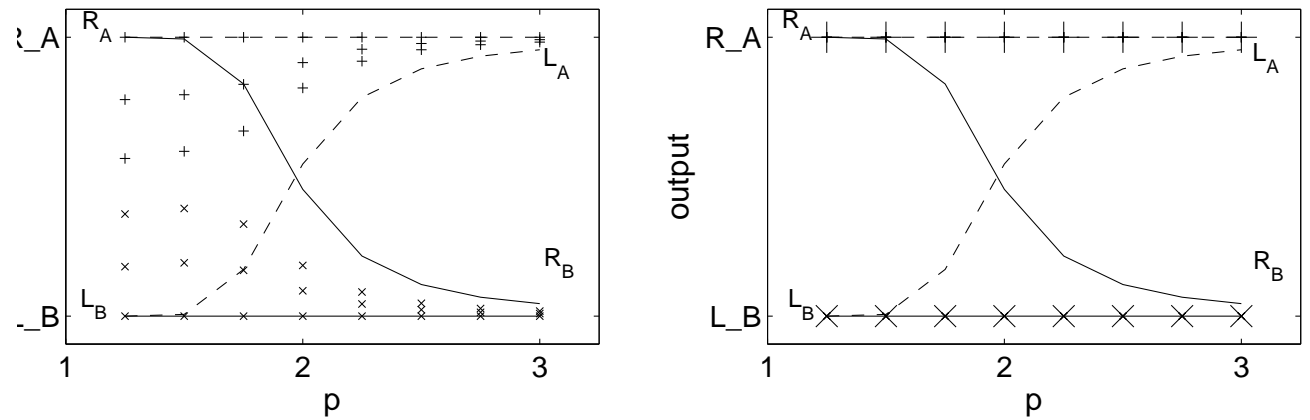

Behind-averse, full reference distribution

Behind-averse, aggregate reference point
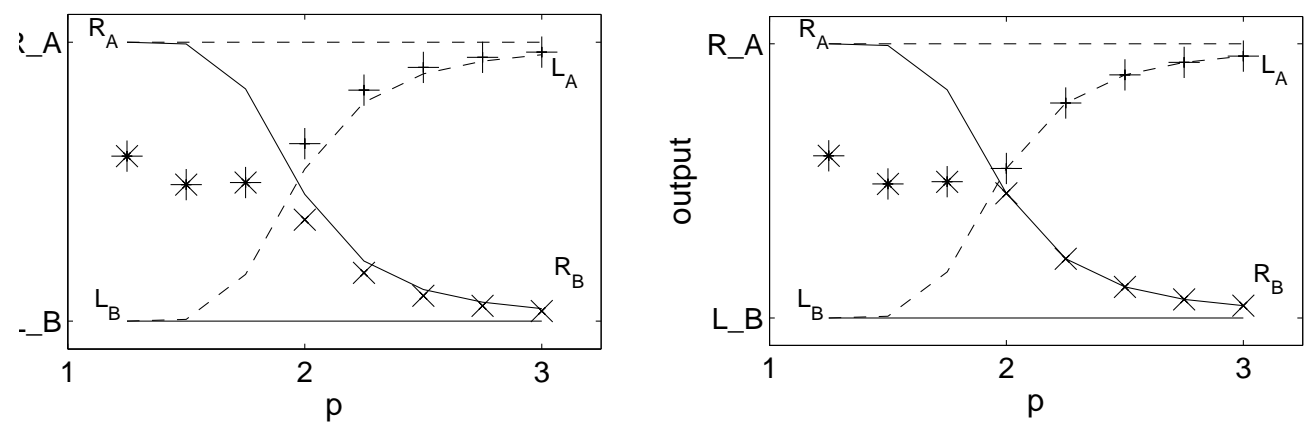

Note. The parameters are: $k=6, \alpha=\beta=2, n_{A}=n_{B}=3$.

We make the following observations from Figure B-5. For large values of $p$, the numerical solutions are similar to the top plots from Figures 2 and 3 with diverse reference groups. For small values of $p$, our numerical results look like the bottom plots from Figures 2 and 3 with uniform reference groups, focusing on only one section. Indeed when $p$ is very small, the strategy sets $\left[L_{B}, R_{B}\right]$ and $\left[L_{A}, R_{A}\right]$ overlap, mimicking a scenario with one uniform type. This numerical study suggests that the structure of our equilibria described in Propositions 1-4 remains valid for a wide range of value functions and that the equilibrium outcomes obtained with a quadratic value function are representative of those obtained with other value functions. 


\section{Acknowledgments}

The authors would like to thank Serguei Netessine, the Associate Editor, the referees, and the seminar participants at Duke, Georgetown, Georgia Tech, Harvard, Kellogg, Penn State, and Stanford for their insightful comments, which have significantly improved this paper. Thanks also to Noah Goldstein and Beril Toktay for pointing out useful references.

\section{References}

Aflaki, S., I. Popescu. 2010. Managing satisfaction in relationships over time. INSEAD Working Paper.

Andreoni, J. 1990. Impure altruism and donations to public goods: A theory of warm-glow giving. Econ. J. 100(401) 464-477.

Avcı, B., Z. Loutfi, J. Mihm, E. Belavina, S. Keck. 2012. Comparison as incentive: Newsvendor comparison in a social context. Prod. $E S$ Oper. Management Forthcoming.

Ayres, I., S. Raseman, A. Shih. 2012. Evidence from two large field experiments that peer comparison feedback can reduce residential energy usage. J. Law Econ. Organ. Forthcoming.

Azmat, G., N. Iriberri. 2010. The importance of relative performance feedback information: Evidence from a natural experiment using high school students. J. Public Econ. 94(7-8) 435-452.

Bandiera, O., I. Barankay, I. Rasul. 2012. Team incentives: Evidence from a field experiment. J. Eur. Econ. Ass. Forthcoming.

Barankay, I. 2012. Rank incentives: Evidence from a randomized workplace experiment. University of Pennsylvania Working Paper.

Baucells, M., R. K. Sarin. 2012. Engineering Happiness. University of California Press.

Blanes i Vidal, J., M. Nossol. 2011. Tournaments without prizes: Evidence from personnel records. Management Sci. 57(10) 1721-1736.

Blanton, H., B. P. Buunk, F. X. Gibbons, H. Kuyper. 1999. When better-than-others compare upward: Choice of comparison and comparative evaluation as independent predictors of academic performance. J. Pers. Soc. Psychol. 76(3) 420-430.

Bolton, G. E., A. Ockenfels. 2000. ERC: A theory of equity, reciprocity, and competition. Amer. Econ. Rev. 90(1) 166-193.

Bond, R. 2005. Group size and conformity. Group Process Interg. 8(4) 331-354.

Brewer, M. B., J. G. Weber. 1994. Self-evaluation effects of interpersonal versus intergroup social comparison. J. Pers. Soc. Psychol. 66(2) 268-275.

Buunk, B. P., R. L. Collins, S. E. Taylor, N. W. VanYperen, G. A Dakof. 1990. The affective consequences of social comparison: Either direction has its ups and downs. J. Pers. Soc. Psychol. 59(6) 1238-1249.

Caro, F., V. Martinez-de Albeniz. 2012. Product and price competition with satiation effects. Management Sci. 58(7) 1357-1373. 
Carrell, S. E., B. I Sacerdote, J. E. West. 2013. From natural variation to optimal policy? The Lucas critique meets peer effects. Econometrica Forthcoming.

Charness, G., M. Rabin. 2002. Understanding social preferences with simple tests. Quart. J. Econ. 117(3) $817-869$.

Collins, R.L. 1996. For better or worse: The impact of upward social comparison on self-evaluations. Psychol. Bull. 119(1) 51-69.

Cui, T. H., J. S. Raju, Z. J. Zhang. 2007. Fairness and channel coordination. Management Sci. 53(8) $1303-1314$.

Delfgaauw, J., J. Dur, J. Sol, W. Verbeke. 2012. Tournament incentives in the field: Gender differences in the workplace. J. Labor Econ. Forthcoming.

DellaVigna, S., U. Malmendier. 2006. Paying not to go to the gym. Amer. Econ. Rev. 96(3) pp. 694-719.

Delmas, M., N. Lessem. 2012. Saving power to conserve your reputation? UCLA Working Paper.

Duflo, E., P. Dupas, M. Kremer. 2011. Peer effects, teacher incentives, and the impact of tracking: Evidence from a randomized evaluation in Kenya. Amer. Econ. Rev. 101(5) 17391774.

Falk, A., A. Ichino. 2006. Clean evidence on peer effects. J. Labor Econ. 24(1) 39-57.

Fehr, E., K. M. Schmidt. 1999. A theory of fairness, competition, and cooperation. Quart. J. Econ. 114(3) $817-868$.

Festinger, L. 1954. A theory of social comparison processes. Hum. Relat. 7 117-140.

Fudenberg, D., J. Tirole. 2000. Game Theory. MIT Press. 7th printing.

Gale, D., H. Nikaidô. 1965. The Jacobian matrix and global univalence of mappings. Mathematische Annalen $\mathbf{1 5 9}(2) 81-93$.

Hardy, G., J. E. Littlewood, G. Pólya. 1952. Inequalities. 2nd ed. Cambridge University Press, New York, NY.

Harsanyi, J. C., R. Selten. 1988. A General Theory of Equilibrium Selection in Games. MIT Press, Cambridge, MA.

Hossain, T., J. A. List. 2012. The behavioralist visits the factory: Increasing productivity using simple manipulations. Management Sci. 58(12) 2151-2167.

Kahneman, D., A. Tversky. 1979. Prospect theory: An analysis of decision under risk. Econometrica 47(2) $263-292$.

Kajii, A., S. Morris. 1997. The robustness of equilibria to incomplete information. Econometrica 65(6) 1283-1309.

Kandel, E., E. P. Lazear. 1992. Peer pressure and partnerships. J. Political Econ. 100(4) 801-817. 
Kojima, F. 2006. Risk-dominance and perfect foresight dynamics in n-player games. J. Econ. Theory 128(1) 255-273.

Kuhnen, C. M., A. Tymula. 2012. Feedback, self-esteem, and performance in organizations. Management Sci. 58(1) 94-113.

Kukushkin, N. S. 2004. A fixed point theorem for decreasing mappings. Econ. Lett. 46(1) 23-26.

Lazear, E. P., S. Rosen. 1981. Rank-order tournaments as optimum labor contracts. J. Political Econ. 89(5) $841-864$.

Levine, D. K. 1998. Modeling altruism and spitefulness in experiments. Rev. Econ. Dynam. 1(3) 593-622.

Lewis, M. A., C. Neighbors. 2008. Social norms approaches using descriptive drinking norms education: A review of the research on personalized normative feedback. J. Amer. Coll. Health 54(4) 213-218.

Loch, C. H., Y. Wu. 2008. Social preferences and supply chain performance: An experimental study. Management Sci. 54(11) 1835-1849.

Lockwood, P., C. H. Jordan, Z. Kunda. 2002. Motivation by positive or negative role models: Regulatory focus determines who will best inspire us. J. Pers. Soc. Psychol. 83(4) 854-864.

Mas, A., E. Moretti. 2009. Peers at work. Amer. Econ. Rev. 99(1) 112-145.

McCardle, K. F., K. Rajaram, C. S. Tang. 2009. A decision analysis tool for evaluating fundraising tiers. Decision Anal. 6(1) 4-13.

Nasiry, J., I. Popescu. 2011. Dynamic pricing with loss-averse consumers and peak-end anchoring. Oper. Res. 59(6) 1361-1368.

Netessine, S., C. S. Tang. 2009. Operations Management Models with Consumer-Driven Demand. Springer International Series in Operations Research \& Management Science, Springer.

Netessine, S., V. Yakubovich. 2012. The Darwinian workplace. Harvard Bus. Rev. 1-4May.

Popescu, I, Y. Wu. 2007. Dynamic pricing strategies with reference effects. Oper. Res. 55(3) 413-429.

Rosen, S. 1986. Prizes and incentives in elimination tournaments. Amer. Econ. Rev. 76(4) 701-715.

Schultz, P. W. 1998. Changing behavior with normative feedback interventions: A field experiment on curbside recycling. Basic Appl. Soc. Psych. 21(1) 25-36.

Schultz, P. W., J. M. Nolan, R. B. Cialdini, N. J. Goldstein, V. Griskevicius. 2007. The constructive, destructive, and reconstructive power of social norms. Psychol. Sci. 18(5) 429-434.

Shen, Z. J., X. Su. 2007. Customer behavior modeling in revenue management and auctions: A review and new research opportunities. Prod. E Oper. Management 16(6) 713-728.

Siemsen, E., S. Balasubramanian, A. Roth. 2007. Incentives that induce task-related effort, helping and knowledge sharing in workgroups. Management Sci. 53(10) 1533-1550. 
Stevenson, H. W., S. Y. Lee, C. Chen, J. W. Stigler, C. C. Hsu, S. Kitamura, G. Hatano. 1990. Context of achievements: A study of American, Chinese, and Japanese students. Monographs of the Society for Research in Child Development 55(1-2) 1-119.

Suls, J., L. Wheeler. 2000. Handbook of Social Comparison: Theory and Research. The Springer Series in Social Clinical Psychology, Springer.

Tereyagoglu, N., S. Veeraraghavan. 2012. Selling to conspicuous consumers: Pricing, production and sourcing decisions. Management Sci. 58(12) 2168-2189.

Thaler, R. H., S. Benartzi. 2004. Save more tomorrow: Using behavioral economics to increase employee saving. J. Political Econ. 112(S1) pp. S164-S187.

Thaler, R. H., C. R. Sunstein. 2008. Nudge. Improving Decisions about Health, Wealth, and Happiness. Yale University Press.

Veeraraghavan, S., L. Debo. 2011. Herding in queues with waiting costs: Rationality and regret. Manufacturing 83 Service Oper. Management 13(3) 329-346.

Vives, X. 1999. Oligopoly Pricing: Old Ideas and New Tools. MIT Press, Cambridge, MA.

Wills, T. A. 1981. Downward comparison principles in social psychology. Psychol. Bull. 90(2) 245-271.

Wood, J. V. 1989. Theory and research concerning social comparisons of personal attributes. Psychol. Bull. 106(2) 231-248. 


\section{Electronic Companion for \\ Optimal Design of Social Comparison Effects: Setting Reference Groups and Reference Points}

Guillaume Roels

UCLA Anderson School of Management, 110 Westwood Plaza, Los Angeles, CA 90095, USA, groels@anderson.ucla.edu Xuanming $\mathrm{Su}$

The Wharton School, University of Pennsylvania, 3730 Walnut Street, Philadelphia, PA 19104, USA, xuanming@wharton.upenn.edu 
Table EC.1 Outputs and utilities with quadratic value functions $V_{A}(x+k)=r_{A}+p x-q x^{2} / 2$ and $V_{B}(x)=r_{B}+p x-q x^{2} / 2$, with $q>0$ when $L_{A}^{\alpha}=L_{A}^{\beta} \geq \max \left\{R_{B}^{\alpha}, R_{B}^{\beta}\right\}$.

\begin{tabular}{|c|c|c|c|}
\hline Game & Type & Heterogeneous group of size $n=n_{A}+n_{B}$ & Homogeneous group of size $n_{\theta}, \theta=A, B$ \\
\hline \multirow[t]{2}{*}{$\beta f$} & $A$ & $\begin{array}{c}x_{A}^{(\beta f 2)}=k+\frac{p}{q}+\frac{\beta}{q}\left(\frac{n_{A}-1}{2}\right) \\
U_{A}^{(\beta f 2)}=r_{A}+\frac{p^{2}}{2 q}-\frac{\beta^{2}}{2 q}\left(\frac{n_{A}-1}{2}\right)^{2}\end{array}$ & $\begin{array}{c}x_{A}^{(\beta f 1)}=k+\frac{p}{q}+\frac{\beta}{q}\left(\frac{n_{A}-1}{2}\right) \\
U_{A}^{(\beta f 1)}=r_{A}+\frac{p^{2}}{2 q}-\frac{\beta^{2}}{2 q}\left(\frac{n_{A}-1}{2}\right)^{2}\end{array}$ \\
\hline & $B$ & $\begin{array}{c}x_{B}^{(\beta f 2)}=\frac{p}{q}+\frac{\beta}{q}\left(n_{A}+\frac{n_{B}-1}{2}\right) \\
U_{B}^{(\beta f 2)}=r_{B}+\frac{p^{2}}{2 q}-\frac{\beta^{2}}{2 q}\left(\frac{n}{2}+\frac{n_{A}-1}{2}\right)^{2}-\beta n_{A}\left(k-\frac{\beta}{q} \frac{n}{2}\right)\end{array}$ & $\begin{array}{c}x_{B}^{(\beta f 1)}=\frac{p}{q}+\frac{\beta}{q}\left(\frac{n_{B}-1}{2}\right) \\
U_{B}^{(\beta f 1)}=r_{B}+\frac{p^{2}}{2 q}-\frac{\beta^{2}}{2 q}\left(\frac{n_{B}-1}{2}\right)^{2}\end{array}$ \\
\hline \multirow[t]{2}{*}{$\beta a$} & $A$ & $\begin{array}{c}x_{A}^{(\beta a 2)}=k+\frac{p}{q} \\
U_{A}^{(\beta a 2)}=r_{A}+\frac{p^{2}}{2 q}\end{array}$ & $\begin{array}{c}x_{A}^{(\beta a 1)}=k+\frac{p}{q}+\frac{\beta}{q}\left(\frac{n_{A}-1}{2}\right) \\
U_{A}^{(\beta a 1)}=r_{A}+\frac{p^{2}}{2 q}-\frac{\beta^{2}}{2 q}\left(\frac{n_{A}-1}{2}\right)^{2}\end{array}$ \\
\hline & $B$ & $\begin{array}{c}x_{B}^{(\beta a 2)}=\frac{p}{q}+\frac{\beta}{q}(n-1) \\
U_{B}^{(\beta a 2)}=r_{B}+\frac{p^{2}}{2 q}-\frac{\beta^{2}}{2 q}(n-1)^{2}-\beta n_{A}\left(k-\frac{\beta}{q}(n-1)\right)\end{array}$ & $\begin{array}{c}x_{B}^{(\beta a 1)}=\frac{p}{q}+\frac{\beta}{q}\left(\frac{n_{B}-1}{2}\right) \\
U_{B}^{(\beta a 1)}=r_{B}+\frac{p^{2}}{2 q}-\frac{\beta^{2}}{2 q}\left(\frac{n_{B}-1}{2}\right)^{2}\end{array}$ \\
\hline \multirow[t]{2}{*}{$\alpha f$} & $A$ & $\begin{array}{c}x_{A, i}^{(\alpha f 2)}=k+\frac{p}{q}+\frac{\alpha}{q}\left(n_{B}+i-1\right) \\
U_{A, i}^{(\alpha f 2)}=r_{A}+\frac{p^{2}}{2 q}+\frac{\alpha^{2}}{2 q}\left(n_{B}+i-1\right)+\alpha n_{B} k\end{array}$ & $\begin{array}{c}x_{A, i}^{(\alpha f 1)}=k+\frac{p}{q}+\frac{\alpha}{q}(i-1) \\
U_{A, i}^{(\alpha f 1)}=r_{A}+\frac{p^{2}}{2 q}+\frac{\alpha^{2}}{2 q}(i-1)\end{array}$ \\
\hline & $B$ & $\begin{array}{c}x_{B, i}^{(\alpha f 2)}=\frac{p}{q}+\frac{\alpha}{q}(i-1) \\
U_{B, i}^{(\alpha f 2)}=r_{B}+\frac{p^{2}}{2 q}+\frac{\alpha^{2}}{2 q}(i-1)\end{array}$ & $\begin{array}{c}x_{B, i}^{(\alpha f 1)}=\frac{p}{q}+\frac{\alpha}{q}(i-1) \\
U_{B, i}^{(\alpha f 1)}=r_{B}+\frac{p^{2}}{2 q}+\frac{\alpha^{2}}{2 q}(i-1)\end{array}$ \\
\hline \multirow[t]{2}{*}{$\alpha a$} & $A$ & $\begin{array}{c}x_{A}^{(\alpha a 2)}=k+\frac{p}{q}+\frac{\alpha}{q}(n-1) \\
U_{A}^{(\alpha a 2)}=r_{A}+\frac{p^{2}}{2 q}+\frac{\alpha^{2}}{q}(n-1)\left(n_{B}-\frac{n-1}{2}\right)+\alpha n_{B} k\end{array}$ & $\begin{array}{c}x_{A, i}^{(\alpha a 1)}=k+\frac{p}{q}+\frac{\alpha}{q}\left(n_{A}-1\right) \mathbb{1}_{\left[i>m_{A}\right]} \\
U_{A, i}^{(\alpha a 1)}=r_{A}+\frac{p^{2}}{2 q}+\frac{\alpha^{2}}{q}\left(n_{A}-1\right)\left(m_{A}-\frac{n_{A}-1}{2}\right) \mathbb{1}_{\left[i>m_{A}\right]}\end{array}$ \\
\hline & $B$ & $\begin{array}{c}x_{B}^{(\alpha a 2)}=\frac{p}{q} \\
U_{B}^{(\alpha a 2)}=r_{B}+\frac{p^{2}}{2 q}\end{array}$ & $\begin{array}{c}x_{B, i}^{(\alpha a 1)}=\frac{p}{q}+\frac{\alpha}{q}\left(n_{B}-1\right) \mathbb{1}_{\left[i>m_{B}\right]} \\
U_{B, i}^{(\alpha a 1)}=r_{B}+\frac{p^{2}}{2 q}+\frac{\alpha^{2}}{q}\left(n_{B}-1\right)\left(m_{B}-\frac{n_{B}-1}{2}\right) \mathbb{1}_{\left[i>m_{B}\right]}\end{array}$ \\
\hline
\end{tabular}

Note: $m_{\theta}=n_{\theta} / 2$ if $n_{\theta}$ is even and can be equal to either $\left(n_{\theta}-1\right) / 2$ or $\left(n_{\theta}+1\right) / 2$ if $n_{\theta}$ is odd.

\section{Additional Results for Quadratic Value Functions}

Table EC.1 displays the equilibrium outputs and utilities for all games and all player types when the value function $V(x)$ is quadratic, obtained from Table A-1 in the appendix of the paper. Specifically, we assume that $V_{A}(x+k)=r_{A}+p x-q x^{2} / 2$ and $V_{B}(x)=r_{B}+p x-q x^{2} / 2$, with $q>0$. Accordingly, $L_{A}^{\alpha}=L_{A}^{\beta}=p / q+k, L_{B}^{\alpha}=L_{B}^{\beta}=p / q, R_{A}^{\alpha}(n)=p / q+\alpha(n-1) / q+k, R_{B}^{\alpha}(n)=$ $p / q+\alpha(n-1) / q, R_{A}^{\beta}(n)=p / q+\beta(n-1) / q+k$, and $R_{B}^{\beta}(n)=p / q+\beta(n-1) / q$. With a quadratic value function, the condition that strategy sets do not overlap is equivalent to requiring that $k \geq \max \{\alpha, \beta\}(n-1) / q$.

Lemma EC.1 (Total Output). When $V(x)$ is quadratic,

$$
\sum_{i=1}^{n_{A}} x_{A, i}^{(\alpha a 2)}+\sum_{i=1}^{n_{B}} x_{B, i}^{(\alpha a 2)} \leq \sum_{i=1}^{n_{A}} x_{A, i}^{(\alpha f 2)}+\sum_{i=1}^{n_{B}} x_{B, i}^{(\alpha f 2)} \text { and } \sum_{i=1}^{n_{A}} x_{A, i}^{(\beta a 2)}+\sum_{i=1}^{n_{B}} x_{B, i}^{(\beta a 2)} \geq \sum_{i=1}^{n_{A}} x_{A, i}^{(\beta f 2)}+\sum_{i=1}^{n_{B}} x_{B, i}^{(\beta f 2)}
$$

if and only if $n_{A} \leq n_{B}$; and

$$
\sum_{i=1}^{2 n} x_{i}^{[\alpha a 2]}=\sum_{i=1}^{2 n} x_{i}^{[\alpha a 1]}=\sum_{i=1}^{2 n} x_{i}^{[\alpha f 2]}=\sum_{i=1}^{2 n} x_{i}^{[\alpha f 1]} \text { and } \sum_{i=1}^{2 n} x_{i}^{[\beta a 2]}=\sum_{i=1}^{2 n} x_{i}^{[\beta a 1]}=\sum_{i=1}^{2 n} x_{i}^{[\beta f 2]}=\sum_{i=1}^{2 n} x_{i}^{[\beta f 1]} .
$$


Proof. For all $\xi=\alpha, \beta, r=a, f$, define $s^{(\xi r 2)}=\sum_{i=1}^{n_{A}} x_{A, i}^{(\xi r 2)}+\sum_{i=1}^{n_{B}} x_{B, i}^{(\xi r 2)}$. From Table EC.1, we obtain that $s^{(\beta f 2)}=n \frac{p}{q}+n_{A} k+\frac{n}{2} \frac{\beta}{q}(n-1), s^{(\beta a 2)}=n \frac{p}{q}+n_{A} k+n_{B} \frac{\beta}{q}(n-1), s^{(\alpha f 2)}=n \frac{p}{q}+n_{A} k+$ $\frac{n}{2} \frac{\alpha}{q}(n-1)$ and $s^{(\alpha a 2)}=n \frac{p}{q}+n_{A} k+n_{A} \frac{\alpha}{q}(n-1)$. Comparing these sums yields the first result.

Next, define $s_{\theta}^{(\xi r 1)}=\sum_{i=1}^{n} x_{\theta, i}^{[\xi r 1]}$ for all $\xi=\alpha, \beta, r=a, f, \theta=A, B$. From Table EC.1, after replacing $n_{A}$ and $n_{B}$ with $n$, we obtain $s_{\theta}^{(\alpha f 1)}=s_{\theta}^{(\alpha a 1)}=n\left(k_{\theta}+\frac{p}{q}+\frac{\alpha}{q}\left(\frac{n}{2}-\frac{1}{2}\right)\right)$ and $s_{\theta}^{(\beta f 1)}=s_{\theta}^{(\beta a 1)}=$ $n\left(k_{\theta}+\frac{p}{q}+\frac{\beta}{q}\left(\frac{n}{2}-\frac{1}{2}\right)\right)$ for $\theta=A, B$, with $k_{A}=k$ and $k_{B}=0$. Comparing $2 s^{(\xi r 2)}$, with $n_{A}$ and $n_{B}$ replaced with $n / 2$, to $s_{A}^{(\xi r 1)}+s_{B}^{(\xi r 1)}$ for all $\xi=\alpha, \beta$ and $r=a, f$ yields the second result.

LEMMA EC.2. When $V(x)$ is quadratic and $n_{A}=n_{B}=n / 2, s_{l}^{(\alpha a 2)} \leq s_{l}^{(\alpha f 2)}$ and $s_{l}^{(\beta f 2)} \leq s_{l}^{(\beta a 2)}$ for all $l \leq n$, in which $s_{l}^{(\xi r 2)}=\sum_{i=1}^{l} x_{i}^{(\xi r 2)}$ for all $\xi=\alpha, \beta, r=a, f$, and $l=1, \ldots, n$.

Proof. Note that $\mathbf{x}^{(\xi r 2)}=\left(\mathbf{x}_{B}^{(\xi r 2)}, \mathbf{x}_{A}^{(\xi r 2)}\right)$ is sorted in ascending order for any $\xi=\alpha, \beta$, and $r=$ $a, f$. From Table EC.1, we obtain:

- $s_{l}^{(\beta f 2)}=\sum_{i=1}^{l}\left(\frac{p}{q}+\frac{\beta}{q}\left(\frac{n}{2}+\frac{n / 2-1}{2}\right)\right)=l \frac{p}{q}+l \frac{\beta}{q}\left(\frac{3 n}{4}-\frac{1}{2}\right)$ if $l \leq n / 2$

- $s_{l}^{(\beta f 2)}=s_{n / 2}^{(\beta f 2)}+\sum_{i=1}^{l-n / 2}\left(k+\frac{p}{q}+\frac{\beta}{q}\left(\frac{n / 2-1}{2}\right)\right)=\left(l-\frac{n}{2}\right) k+l \frac{p}{q}+\frac{\beta}{q} \frac{n^{2}}{4}+l \frac{\beta}{q}\left(\frac{n}{4}-\frac{1}{2}\right)$ if $n / 2<l \leq n$

- $s_{l}^{(\beta a 2)}=\sum_{i=1}^{l}\left(\frac{p}{q}+\frac{\beta}{q}(n-1)\right)=l \frac{p}{q}+l \frac{\beta}{q}(n-1)$ if $l \leq n / 2$

- $s_{l}^{(\beta a 2)}=s_{n / 2}^{(\beta a 2)}+\sum_{i=1}^{l-n / 2}\left(k+\frac{p}{q}\right)=\left(l-\frac{n}{2}\right) k+l \frac{p}{q}+\frac{n}{2} \frac{\beta}{q}(n-1)$ if $n / 2<l \leq n$

- $s_{l}^{(\alpha f 2)}=\sum_{i=1}^{l}\left(\frac{p}{q}+\frac{\alpha}{q}(i-1)\right)=l \frac{p}{q}+\frac{l}{2} \frac{\alpha}{q}(l-1)$ if $l \leq n / 2$

- $s_{l}^{(\alpha f 2)}=s_{n / 2}^{(\alpha f 2)}+\sum_{i=1}^{l-n / 2}\left(k+\frac{p}{q}+\frac{\alpha}{q}\left(\frac{n}{2}+i-1\right)\right)=\left(l-\frac{n}{2}\right) k+l \frac{p}{q}+\frac{l}{2} \frac{\alpha}{q}(l-1)$ if $n / 2<l \leq n$

- $s_{l}^{(\alpha a 2)}=\sum_{i=1}^{l} \frac{p}{q}=l \frac{p}{q}$ if $l \leq n / 2$

- $s_{l}^{(\alpha a 2)}=s_{n / 2}^{(\alpha a 2)}+\sum_{i=1}^{l-n / 2}\left(k+\frac{p}{q}+\frac{\alpha}{q}(n-1)\right)=\left(l-\frac{n}{2}\right) k+l \frac{p}{q}+\frac{\alpha}{q}\left(l-\frac{n}{2}\right)(n-1)$ if $n / 2<l \leq n$.

We then find that $s_{l}^{(\alpha a 2)} \leq s_{l}^{(\alpha f 2)}$ if and only if $0 \leq \frac{l}{2} \frac{\alpha}{q}(l-1)$ when $l \leq n / 2$, which is always true. And if and only if $\frac{\alpha}{q}\left(l-\frac{n}{2}\right)(n-1) \leq \frac{l}{2} \frac{\alpha}{q}(l-1)$ when $l>n / 2$, i.e., if and only if $l^{2} / 2-l(n-1 / 2)+$ $n(n-1) / 2 \geq 0$. The right-hand side is a quadratic equation in $l$, with roots at $n$ and $n-1$. Since $l$ is integer, this condition is satisfied for all $l$. Hence, $s_{l}^{(\alpha a 2)} \leq s_{l}^{(\alpha f 2)}$ for all $l \leq n$.

Moreover, we find that $s_{l}^{(\beta f 2)} \leq s_{l}^{(\beta a 2)}$ if and only if $l \frac{\beta}{q}\left(\frac{3 n}{4}-\frac{1}{2}\right) \leq l \frac{\beta}{q}(n-1)$ when $l \leq n / 2$, which is always true given that $n \geq 2$. And if and only if $\frac{\beta}{q} \frac{n^{2}}{4}+l \frac{\beta}{q}\left(\frac{n}{4}-\frac{1}{2}\right) \leq \frac{n}{2} \frac{\beta}{q}(n-1)$ when $l>n / 2$, which is always true given that $l \leq n$. Hence, $s_{l}^{(\beta f 2)} \leq s_{l}^{(\beta a 2)}$ for all $l$. This shows the desired result.

LEMma EC.3. When $V(x)$ is quadratic, $\sum_{i=1}^{l} x_{i}^{[\beta r 1]} \leq \sum_{i=1}^{l} x_{i}^{[\beta r 2]}$ and $\sum_{i=1}^{l} x_{i}^{[\alpha r 1]} \geq \sum_{i=1}^{l} x_{i}^{[\alpha r 2]}$ for all $l \leq 2 n$, for any $r=a, f$.

Proof. $\quad$ Fix $\xi=\alpha, \beta$ and $r=a, f$. Define $s_{l}^{(\xi r 1)}=\sum_{i=1}^{l} x_{i}^{[\xi r 1]}, l=1, \ldots, 2 n$. Closed-form expressions for $x_{\theta, i}^{[\xi r 1]}$ and $x_{\theta, i}^{[\xi r 2]}$ can be obtained from Table EC.1, with $\theta=A, B$, by making the following transformations: (i) $x_{\theta, i}^{[\xi r 2]}=x_{\theta,\left[\frac{i}{2}\right]}^{(\xi r 2)}$ with $n_{A}=n / 2$ and $n_{B}=n / 2$ and (ii) $x_{\theta, i}^{[\xi r 1]}=x_{\theta, i}^{(\xi r 1)}$ with $n_{A}=n$ and $n_{B}=n$. Note that $\mathbf{x}^{[\xi r 1]}=\left(\mathbf{x}_{B}^{[\xi r 1]}, \mathbf{x}_{A}^{[\xi r 1]}\right)$ is sorted in ascending order, and so is $\mathbf{x}^{[\xi r 2]}$. From Table EC.1, we obtain: 
- $s_{l}^{(\beta f 1)}=\sum_{i=1}^{l}\left(\frac{p}{q}+\frac{\beta}{q}\left(\frac{n-1}{2}\right)\right)=l \frac{p}{q}+l \frac{\beta}{2 q}(n-1)$ if $l \leq n$

- $s_{l}^{(\beta f 1)}=s_{n}^{(\beta f 1)}+\sum_{i=1}^{l-n}\left(k+\frac{p}{q}+\frac{\beta}{q}\left(\frac{n-1}{2}\right)\right)=(l-n) k+l \frac{p}{q}+\frac{l}{2} \frac{\beta}{q}(n-1)$ if $n<l \leq 2 n$

- $s_{l}^{(\beta a 1)}=\sum_{i=1}^{l}\left(\frac{p}{q}+\frac{\beta}{q}\left(\frac{n-1}{2}\right)\right)=l \frac{p}{q}+l \frac{\beta}{2 q}(n-1)$ if $l \leq n$

- $s_{l}^{(\beta a 1)}=s_{n}^{(\beta a 1)}+\sum_{i=1}^{l-n}\left(k+\frac{p}{q}+\frac{\beta}{q}\left(\frac{n-1}{2}\right)\right)=(l-n) k+l \frac{p}{q}+\frac{l}{2} \frac{\beta}{q}(n-1)$ if $n<l \leq 2 n$

- $s_{l}^{(\alpha f 1)}=\sum_{i=1}^{l}\left(\frac{p}{q}+\frac{\alpha}{q}(i-1)\right)=l \frac{p}{q}+\frac{l}{2} \frac{\alpha}{q}(l-1)$ if $l \leq n$

- $s_{l}^{(\alpha f 1)}=s_{n}^{(\alpha f 1)}+\sum_{i=1}^{l-n}\left(k+\frac{p}{q}+\frac{\alpha}{q}(i-1)\right)=(l-n) k+l \frac{p}{q}+\frac{n}{2} \frac{\alpha}{q}(n-1)+\frac{(l-n)}{2} \frac{\alpha}{q}(l-n-1)$ if

$n<l \leq 2 n$

- $s_{l}^{(\alpha a 1)}=\sum_{i=1}^{l}\left(\frac{p}{q}+\frac{\alpha}{q}(n-1) \mathbb{1}_{\left[i>\frac{n}{2}\right]}\right)=l \frac{p}{q}+\left(l-\frac{n}{2}\right)^{+} \frac{\alpha}{q}(n-1)$ if $l \leq n$

- $s_{l}^{(\alpha a 1)}=s_{n}^{(\alpha a 1)}+\sum_{i=1}^{l-n}\left(k+\frac{p}{q}+\frac{\alpha}{q}(n-1) \mathbb{1}_{\left[i>\frac{n}{2}\right]}\right)=(l-n) k+l \frac{p}{q}+\frac{n}{2} \frac{\alpha}{q}(n-1)+\left(l-\frac{3 n}{2}\right)^{+} \frac{\alpha}{q}(n-1)$

if $n<l \leq 2 n$.

We obtain that $s_{l}^{(\beta f 1)} \leq \sum_{i=1}^{l} x_{i}^{[\beta f 2]}$ because (i) when $l \leq n, l \frac{\beta}{2 q}(n-1) \leq l \frac{\beta}{q}\left(\frac{3 n}{4}-\frac{1}{2}\right)$ and (ii) when $n<l \leq 2 n, \frac{l}{2} \frac{\beta}{q}(n-1) \leq \frac{\beta}{2 q} n^{2}+l \frac{\beta}{2 q}\left(\frac{n}{2}-1\right)$. Similarly, $s_{l}^{(\beta a 1)} \leq \sum_{i=1}^{l} x_{i}^{[\beta a 2]}$ because (i) when $l \leq n$, $l \frac{\beta}{2 q}(n-1) \leq l \frac{\beta}{q}(n-1)$ and (ii) when $n<l \leq 2 n, \frac{l}{2} \frac{\beta}{q}(n-1) \leq n \frac{\beta}{q}(n-1)$.

On the other hand, $s_{l}^{(\alpha f 1)} \geq \sum_{i=1}^{l} x_{i}^{[\alpha f 2]}$ because (i) when $\left.l \leq n, \frac{l}{2} \frac{\alpha}{q}(l-1) \geq \frac{\alpha}{q}\left\lfloor\frac{l}{2}\right\rfloor\left(\left\lfloor\frac{l}{2}\right\rfloor-1\right)\right)+(l-$ $\left.2\left\lfloor\frac{l}{2}\right\rfloor\right) \frac{\alpha}{q}\left\lfloor\frac{l}{2}\right\rfloor$ and (ii) when $\left.n<l \leq 2 n, \frac{n}{2} \frac{\alpha}{q}(n-1)+\frac{(l-n)}{2} \frac{\alpha}{q}(l-n-1) \geq \frac{\alpha}{q}\left\lfloor\frac{l}{2}\right\rfloor\left(\left\lfloor\frac{l}{2}\right\rfloor-1\right)\right)+\left(l-2\left\lfloor\frac{l}{2}\right\rfloor\right) \frac{\alpha}{q}\left\lfloor\frac{l}{2}\right\rfloor$. Finally, $s_{l}^{(\alpha a 1)} \geq \sum_{i=1}^{l} x_{i}^{[\alpha a 2]}$ because (i) when $l \leq n,\left(l-\frac{n}{2}\right)^{+} \frac{\alpha}{q}(n-1) \geq 0$ and (ii) when $n<l \leq 2 n$, $\frac{n}{2} \frac{\alpha}{q}(n-1)+\left(l-\frac{3 n}{2}\right)^{+} \frac{\alpha}{q}(n-1) \geq \frac{\alpha}{q}(l-n)(n-1)$.

Lemma EC.4. When $V(x)$ is quadratic,

1. $U_{A, i}^{[\alpha f 2]} \geq U_{A, i}^{[\alpha f 1]}$ and $U_{B, i}^{[\alpha f 2]} \leq U_{B, i}^{[\alpha f 1]}$;

2. $U_{A, i}^{[\alpha a 2]} \geq U_{A, i}^{[\alpha a 1]}$ and $U_{B, i}^{[\alpha a 2]} \leq U_{B, i}^{[\alpha a 1]}$.

Proof. The results follow by comparing the respective entries in Table EC.1 after making the following substitutions: (i) $U_{\theta, i}^{[\xi r 2]}=U_{\theta,\left[\frac{i}{2}\right\rceil}^{(\xi r 2)}$ with $n_{A}=n / 2$ and $n_{B}=n / 2$ and (ii) $U_{\theta, i}^{[\xi r 1]}=U_{\theta, i}^{(\xi r 1)}$ with $n_{A}=n$ and $n_{B}=n$, for $\xi=\alpha, \beta$ and $r=f, p$, and $i=1, \ldots, 2 n$ :

1. $U_{A, i}^{[\alpha f 2]} \geq U_{A, i}^{[\alpha f 1]} \Leftrightarrow \frac{\alpha^{2}}{2 q}\left(\frac{n}{2}+\left\lceil\frac{i}{2}\right\rceil-1\right)+\alpha \frac{n}{2} k \geq \frac{\alpha^{2}}{2 q}(i-1)$, which holds true for all $i \leq n$. And, $U_{B, i}^{[\alpha f 2]} \leq U_{B, i}^{[\alpha f 1]} \Leftrightarrow \frac{\alpha^{2}}{2 q}\left(\left\lceil\frac{i}{2}\right\rceil-1\right) \leq \frac{\alpha^{2}}{2 q}(i-1)$, which is always true.

2. $U_{A, i}^{[\alpha a 2]} \geq U_{A, i}^{[\alpha a 1]} \Leftrightarrow \frac{\alpha^{2}}{q}(n-1)\left(\frac{n}{2}-\frac{n-1}{2}\right)+\alpha \frac{n}{2} k \geq \frac{\alpha^{2}}{q}(n-1)\left(\frac{n}{2}-\frac{n-1}{2}\right) \mathbb{1}_{\left[i>\frac{n}{2}\right]}$, which is always true. And, $U_{B, i}^{[\alpha a 2]} \leq U_{B, i}^{[\alpha a 1]} \Leftrightarrow 0 \leq \frac{\alpha^{2}}{q}(n-1)\left(\frac{n}{2}-\frac{n-1}{2}\right) \mathbb{1}_{\left[i>\frac{n}{2}\right]}$, which is always true.

Lemma EC.5. When $V(x)$ is quadratic,

1. $U_{A, i}^{(\alpha a 2)} \geq U_{A, i}^{(\alpha f 2)}$ for all $i$ if $n_{A} \leq n / 2$;

2. $U_{B, i}^{(\beta a 2)} \geq U_{B, i}^{(\beta f 2)}$ for all $i$ if $n_{A} \geq n / 2$.

Proof. 1. From Table EC.1, we obtain that $U_{A}^{(\alpha a 2)} \geq U_{A, n_{A}}^{(\alpha f 2)}$ if and only if $(n-1)\left(n_{B}-\frac{n-1}{2}\right) \geq$ $(n-1) / 2$ or equivalently, if and only if $n_{A} \leq n / 2$. 
2. From Table EC.1, we obtain that $U_{B}^{(\beta a 2)} \geq U_{B}^{(\beta f 2)}$ if and only if $-(n-1)^{2} / 2+n_{A}(n-1) \geq$ $-\left(n+n_{A}-1\right)^{2} / 8+n_{A} n / 2$ or equivalently, if and only if $n_{A}^{2}+2 n_{A}(3 n-5)-3(n-1)^{2} \geq 0$. The righthand side is a quadratic equation in $n_{A}$, equal to $-3(n-2)^{2}<0$ when $n_{A}=1$ and to $n^{2} / 4+n-3 \geq 0$ when $n_{A}=n / 2$. Hence, there exists a root $\hat{n}$ between 0 and $n / 2$.

LEmma EC.6. When $V(x)$ is quadratic,

$$
\begin{aligned}
& \sum_{i=1}^{n_{A}} U_{A, i}^{[\alpha f 2]}+\sum_{i=1}^{n_{B}} U_{B, i}^{[\alpha f 2]}=\sum_{i=1}^{n_{A}} U_{A, i}^{[\alpha a 2]}+\sum_{i=1}^{n_{B}} U_{B, i}^{[\alpha a 2]} \geq \sum_{i=1}^{n_{A}} U_{A, i}^{[\alpha f 1]}+\sum_{i=1}^{n_{B}} U_{B, i}^{[\alpha f 1]}=\sum_{i=1}^{n_{A}} U_{A, i}^{[\alpha a 1]}+\sum_{i=1}^{n_{B}} U_{B, i}^{[\alpha a 1]} \\
& \sum_{i=1}^{n_{A}} U_{A, i}^{[\beta a 1]}+\sum_{i=1}^{n_{B}} U_{B, i}^{[\beta a 1]}=\sum_{i=1}^{n_{A}} U_{A, i}^{[\beta f 1]}+\sum_{i=1}^{n_{B}} U_{B, i}^{[\beta f 1]} \geq \sum_{i=1}^{n_{A}} U_{A, i}^{[\beta a 2]}+\sum_{i=1}^{n_{B}} U_{B, i}^{[\beta a 2]} \geq \sum_{i=1}^{n_{A}} U_{A, i}^{[\beta f 2]}+\sum_{i=1}^{n_{B}} U_{B, i}^{[\beta f 2]} .
\end{aligned}
$$

Proof. The result follows from comparing the respective entries in Table EC.1 after making the following substitutions: (i) $U_{\theta, i}^{[\xi r 2]}=U_{\theta,\left[\frac{i}{2}\right\rceil}^{(\xi r 2)}$ with $n_{A}=n / 2$ and $n_{B}=n / 2$ and (ii) $U_{\theta, i}^{[\xi r 1]}=U_{\theta, i}^{(\xi r 1)}$ with $n_{A}=n$ and $n_{B}=n$, for any $\xi=\alpha, \beta$ and $r=f, p$, and $i=1, \ldots, 2 n$. We then obtain:

$$
\begin{aligned}
& \sum_{i=1}^{n_{A}} U_{A, i}^{[\beta f 2]}+\sum_{i=1}^{n_{B}} U_{B, i}^{[\beta f 2]}=n\left(r_{A}+\frac{p^{2}}{2 q}-\frac{\beta^{2}}{2 q}\left(\frac{\frac{n}{2}-1}{2}\right)^{2}\right)+n\left(r_{B}+\frac{p^{2}}{2 q}-\frac{\beta^{2}}{2 q}\left(\frac{\frac{3 n}{2}-1}{2}\right)^{2}-\beta \frac{n}{2}\left(k-\frac{\beta}{q} \frac{n}{2}\right)\right) \\
& \sum_{i=1}^{n_{A}} U_{A, i}^{[\beta f 1]}+\sum_{i=1}^{n_{B}} U_{B, i}^{[\beta f 1]}=n\left(r_{A}+\frac{p^{2}}{2 q}-\frac{\beta^{2}}{2 q}\left(\frac{n-1}{2}\right)^{2}\right)+n\left(r_{B}+\frac{p^{2}}{2 q}-\frac{\beta^{2}}{2 q}\left(\frac{n-1}{2}\right)^{2}\right) \\
& \sum_{i=1}^{n_{A}} U_{A, i}^{[\beta a 2]}+\sum_{i=1}^{n_{B}} U_{B, i}^{[\beta a 2]}=n\left(r_{A}+\frac{p^{2}}{2 q}\right)+n\left(r_{B}+\frac{p^{2}}{2 q}-\frac{\beta^{2}}{2 q}(n-1)^{2}-\beta \frac{n}{2}\left(k-\frac{\beta}{q}(n-1)\right)\right) \\
& \sum_{i=1}^{n_{A}} U_{A, i}^{[\beta a 1]}+\sum_{i=1}^{n_{B}} U_{B, i}^{[\beta a 1]}=n\left(r_{A}+\frac{p^{2}}{2 q}-\frac{\beta^{2}}{2 q}\left(\frac{n-1}{2}\right)^{2}\right)+n\left(r_{B}+\frac{p^{2}}{2 q}-\frac{\beta^{2}}{2 q}\left(\frac{n-1}{2}\right)^{2}\right) \\
& \sum_{i=1}^{n_{A}} U_{A, i}^{[\alpha f 2]}+\sum_{i=1}^{n_{B}} U_{B, i}^{[\alpha f 2]}=n\left(r_{A}+\frac{p^{2}}{2 q}+\frac{\alpha^{2}}{2 q}\left(\frac{n}{2}+\frac{1}{2}\left(\frac{n}{2}-1\right)\right)+\alpha \frac{n}{2} k\right)+n\left(r_{B}+\frac{p^{2}}{2 q}+\frac{\alpha^{2}}{2 q} \frac{1}{2}\left(\frac{n}{2}-1\right)\right) \\
& \sum_{i=1}^{n_{A}} U_{A, i}^{[\alpha f 1]}+\sum_{i=1}^{n_{B}} U_{B, i}^{[\alpha f 1]}=n\left(r_{A}+\frac{p^{2}}{2 q}+\frac{\alpha^{2}}{2 q} \frac{(n-1)}{2}\right)+n\left(r_{B}+\frac{p^{2}}{2 q}+\frac{\alpha^{2}}{2 q} \frac{(n-1)}{2}\right) \\
& \sum_{i=1}^{n_{A}} U_{A, i}^{[\alpha a 2]}+\sum_{i=1}^{n_{B}} U_{B, i}^{[\alpha a 2]}=n\left(r_{A}+\frac{p^{2}}{2 q}+\frac{\alpha^{2}}{q}(n-1)\left(\frac{n}{2}-\frac{n-1}{2}\right)+\alpha \frac{n}{2} k\right)+n\left(r_{B}+\frac{p^{2}}{2 q}\right) \\
& \sum_{i=1}^{n_{A}} U_{A, i}^{[\alpha a 1]}+\sum_{i=1}^{n_{B}} U_{B, i}^{[\alpha a 1]}=n\left(r_{A}+\frac{p^{2}}{2 q}+\frac{\alpha^{2}}{q} \frac{(n-1)}{4}\right)+n\left(r_{B}+\frac{p^{2}}{2 q}+\frac{\alpha^{2}}{q} \frac{(n-1)}{4}\right)
\end{aligned}
$$

It is easy to check that $\sum_{i=1}^{n_{A}} U_{A, i}^{[\alpha f 2]}+\sum_{i=1}^{n_{B}} U_{B, i}^{[\alpha f 2]}=\sum_{i=1}^{n_{A}} U_{A, i}^{[\alpha a 2]}+\sum_{i=1}^{n_{B}} U_{B, i}^{[\alpha a 2]}$, that $\sum_{i=1}^{n_{A}} U_{A, i}^{[\alpha f 1]}+$ $\sum_{i=1}^{n_{B}} U_{B, i}^{[\alpha f 1]}=\sum_{i=1}^{n_{A}} U_{A, i}^{[\alpha a 1]}+\sum_{i=1}^{n_{B}} U_{B, i}^{[\alpha a 1]}$ and that $\sum_{i=1}^{n_{A}} U_{A, i}^{[\beta a 1]}+\sum_{i=1}^{n_{B}} U_{B, i}^{[\beta a 1]}=\sum_{i=1}^{n_{A}} U_{A, i}^{[\beta f 1]}+$ $\sum_{i=1}^{n_{B}} U_{B, i}^{[\beta f 1]}$. We find that $\sum_{i=1}^{n_{A}} U_{A, i}^{[\beta a 2]}+\sum_{i=1}^{n_{B}} U_{B, i}^{[\beta a 2]} \geq \sum_{i=1}^{n_{A}} U_{A, i}^{[\beta f 2]}+\sum_{i=1}^{n_{B}} U_{B, i}^{[\beta f 2]}$ if and only if $\beta^{2}(n-1) /(2 q) \geq \beta^{2}\left(n-n^{2} / 8-1 / 2\right) /(2 q)$, which always holds true when $n \geq 2$. Also, $\sum_{i=1}^{n_{A}} U_{A, i}^{[\beta a 1]}+$ $\sum_{i=1}^{n_{B}} U_{B, i}^{[\beta a 1]} \geq \sum_{i=1}^{n_{A}} U_{A, i}^{[\beta a 2]}+\sum_{i=1}^{n_{B}} U_{B, i}^{[\beta a 2]}$ if and only if $-\beta^{2}(n-1)^{2} /(4 q) \geq \beta^{2}(n-1) /(2 q)-k \beta n / 2$, 
which always holds true given that $k \geq \beta(n-1) / q$. Finally, $\sum_{i=1}^{n_{A}} U_{A, i}^{[\alpha a 2]}+\sum_{i=1}^{n_{B}} U_{B, i}^{[\alpha a 2]} \geq \sum_{i=1}^{n_{A}} U_{A, i}^{[\alpha a 1]}+$ $\sum_{i=1}^{n_{B}} U_{B, i}^{[\alpha a 1]}$ if and only if $\alpha^{2}(n-1) /(2 q)+\alpha n k / 2 \geq \alpha^{2}(n-1) /(2 q)$, which is true for all $k \geq 0$. 